\title{
Hopf Bifurcation Analysis for a Semiratio-Dependent Predator-Prey System with Two Delays
}

\author{
Ming Zhao \\ Software College, Pingdingshan University, Pingdingshan 467000, China \\ Correspondence should be addressed to Ming Zhao; zhaomingxh2013@163.com
}

Received 12 August 2013; Accepted 25 August 2013

Academic Editor: Massimiliano Ferrara

Copyright (C) 2013 Ming Zhao. This is an open access article distributed under the Creative Commons Attribution License, which permits unrestricted use, distribution, and reproduction in any medium, provided the original work is properly cited.

\begin{abstract}
This paper is concerned with a semiratio-dependent predator-prey system with nonmonotonic functional response and two delays. It is shown that the positive equilibrium of the system is locally asymptotically stable when the time delay is small enough. Change of stability of the positive equilibrium will cause bifurcating periodic solutions as the time delay passes through a sequence of critical values. The properties of Hopf bifurcation such as direction and stability are determined by using the normal form method and center manifold theorem. Numerical simulations confirm our theoretical findings.
\end{abstract}

\section{Introduction}

It is well known that there are many factors which can affect dynamical properties of predator-prey systems. One of the important factors is the functional response describing the number of prey consumed per predator per unit time for given quantities of prey and predators. Numerous laboratory experiment, and observations have shown that a more suitable general predator-prey system should be based on the "ratio-dependent" theory, especially when predators have to search, share, or compete for food [1-3]. And ratiodependent predator-prey systems have been investigated by many scholars [4-11]. In [4], Zhang and Lu considered the following semi-ratio-dependent predator-prey system with the nonmonotonic functional response

$$
\begin{aligned}
\frac{d x_{1}(t)}{d t}= & x_{1}(t) \\
& \times\left[r_{1}(t)-b_{1}(t) x_{1}(t)-\frac{a_{1}(t) x_{2}(t)}{m^{2}+n x_{1}(t)+x_{1}^{2}(t)}\right], \\
& \frac{d x_{2}(t)}{d t}=x_{2}(t)\left[r_{2}(t)-\frac{a_{2}(t) x_{2}(t)}{x_{1}(t)}\right],
\end{aligned}
$$

where $x_{1}(t)$ and $x_{2}(t)$ denote the densities of the prey and the predator, respectively. $r_{1}(t)$ and $r_{2}(t)$ denote the intrinsic growth rates of the prey and the predator, respectively. $b_{1}(t)$ is the intraspecific competition rate of the prey. $a_{1}(t)$ is the capturing rate of the predator. $a_{2}(t)$ is a measure of the food quality that the prey provided for conversion into predator birth. The predator grows logistically with the carrying capacity $x_{1}(t) / a_{2}(t)$ proportional to the population size of the prey.

$m \neq 0, n \geq 0$, and $a_{i}(t), r_{i}(t), b_{1}(t),(i=1,2)$ are assumed to be continuously positive periodic functions with period $\omega$. Zhang and $\mathrm{Lu}[4]$ established the existence of positive periodic solutions of system (1), and sufficient conditions for the uniqueness and global stability of the positive solutions of system (1) were also obtained by constructing a Lyapunov function.

Time delays of one type or another have been incorporated into predator-prey systems by many researchers since a time delay could cause a stable equilibrium to become unstable and cause the population to fluctuate $[5,6,12-15]$. In [5], Ding et al. incorporated the time delay $\tau(t) \geq 0$ due to negative feedback of the prey into system (1) and got the special case $(n=0)$ of system (1) with time delay 


$$
\begin{gathered}
\frac{d x_{1}(t)}{d t}=x_{1}(t)\left[r_{1}(t)-b_{1}(t) x_{1}(t-\tau(t))-\frac{a_{1}(t) x_{2}(t)}{m^{2}+x_{1}^{2}(t)}\right], \\
\frac{d x_{2}(t)}{d t}=x_{2}(t)\left[r_{2}(t)-\frac{a_{2}(t) x_{2}(t)}{x_{1}(t)}\right] .
\end{gathered}
$$

Ding et al. [5] established the existence of a positive periodic solution for system (2) by using the continuation theorem of coincidence degree theory. Sufficient conditions for the permanence of system (2) were obtained by Li and Yang in [6]. But studies on predator-prey systems not only involve the persistence and the periodic phenomenon but also involve many patterns of other behavior such as global attractivity $[16,17]$ and bifurcation phenomenon [18-21]. Starting from this point, we will study the bifurcation phenomenon and the properties of periodic solutions of the following predatorprey system with two delays:

$$
\begin{gathered}
\frac{d x_{1}(t)}{d t}=x_{1}(t)\left[r_{1}-b_{1} x_{1}\left(t-\tau_{1}\right)-\frac{a_{1} x_{2}(t)}{m^{2}+n x_{1}(t)+x_{1}^{2}(t)}\right], \\
\frac{d x_{2}(t)}{d t}=x_{2}(t)\left[r_{2}-\frac{a_{2} x_{2}\left(t-\tau_{2}\right)}{x_{1}\left(t-\tau_{2}\right)}\right] .
\end{gathered}
$$

Unlike the assumptions in [4-6], we assume that all the parameters of system (3) are positive constants. $\tau_{1} \geq 0$ and $\tau_{2} \geq 0$ are the feedback delays of the prey and the predator, respectively.

The rest of this paper is organized as follows. In Section 2, sufficient conditions are obtained for the local stability of the positive equilibrium and the existence of Hopf bifurcation for possible combinations of the two delays in system (3). In Section 3, we give the formula determining the direction of Hopf bifurcation and the stability of the bifurcating periodic solutions. Finally, numerical simulations supporting the theoretical analysis are also included.

\section{Stability of the Positive Equilibrium and Local Hopf Bifurcations}

It is not difficult to verify that system (2) has at least one positive equilibrium $E^{*}\left(x_{1}^{*}, x_{2}^{*}\right)$, where $x_{2}^{*}=r_{2} x_{1}^{*} / a_{2}$ and $x_{1}^{*}$ is the root of (4)

$$
b_{1} x_{1}^{3}+\left(n b_{1}-r_{1}\right) x_{1}^{2}+\left(\frac{a_{1} r_{2}}{a_{2}}-n r_{1}+m^{2} b_{1}\right) x_{1}-m^{2} r_{1}=0 .
$$

Let $\bar{x}_{1}(t)=x_{1}(t)-x_{1}^{*}, \bar{x}_{2}(t)=x_{2}(t)-x_{2}^{*}$. Dropping the bars for the sake of simplicity, system (2) can be rewritten in the following system:

$$
\begin{gathered}
\frac{d x_{1}(t)}{d t}=a_{11} x_{1}(t)+a_{12} x_{2}(t)+b_{11} x_{1}\left(t-\tau_{1}\right)+f_{1}, \\
\frac{d x_{2}(t)}{d t}=c_{21} x_{1}\left(t-\tau_{2}\right)+c_{22} x_{2}\left(t-\tau_{2}\right)+f_{2},
\end{gathered}
$$

where

$$
\begin{aligned}
& a_{11}=\frac{a_{1} x_{1}^{*} x_{2}^{*}\left(n+2 x_{1}^{*}\right)}{\left(m^{2}+n x_{1}^{*}+\left(x_{1}^{*}\right)^{2}\right)^{2}} \\
& a_{12}=-\frac{a_{1} x_{1}^{*}}{m^{2}+n x_{1}^{*}+\left(x_{1}^{*}\right)^{2}} \text {, } \\
& b_{11}=-b_{1} x_{1}^{*}, \quad c_{21}=\frac{a_{2}\left(x_{2}^{*}\right)^{2}}{\left(x_{1}^{*}\right)^{2}}, \quad c_{22}=-\frac{a_{2} x_{2}^{*}}{x_{1}^{*}}, \\
& f_{1}=a_{13} x_{1}^{2}(t)+a_{14} x_{1}(t) x_{2}(t)+a_{15} x_{1}(t) x_{1}\left(t-\tau_{1}\right) \\
& +a_{16} x_{1}^{2}(t) x_{2}(t)+a_{17} x_{1}^{3}(t)+\cdots \\
& f_{2}=c_{23} x_{1}^{2}\left(t-\tau_{2}\right)+c_{24} x_{1}\left(t-\tau_{2}\right) x_{2}(t) \\
& +c_{25} x_{2}\left(t-\tau_{2}\right) x_{2}(t)+c_{26} x_{1}\left(t-\tau_{2}\right) x_{2}\left(t-\tau_{2}\right) \\
& +c_{27} x_{1}^{3}\left(t-\tau_{2}\right)+c_{28} x_{1}^{2}\left(t-\tau_{2}\right) x_{2}(t) \\
& +c_{29} x_{1}^{2}\left(t-\tau_{2}\right) x_{2}\left(t-\tau_{2}\right)+c_{210} x_{1}\left(t-\tau_{2}\right) \\
& \times x_{2}\left(t-\tau_{2}\right) x_{2}(t)+\cdots, \\
& a_{13}=\frac{a_{1} x_{1}^{*} x_{2}^{*}\left(m^{2}-n^{2}-3 n x_{1}^{*}-3\left(x_{1}^{*}\right)^{2}\right)}{\left(m^{2}+n x_{1}^{*}+\left(x_{1}^{*}\right)^{2}\right)^{3}} \text {, } \\
& a_{14}=\frac{a_{1}\left(\left(x_{1}^{*}\right)^{2}-m^{2}\right)}{\left(m^{2}+n x_{1}^{*}+\left(x_{1}^{*}\right)^{2}\right)^{2}}, \quad a_{15}=-b_{1}, \\
& a_{16}=\frac{a_{1} x_{1}^{*}\left(m^{2}-n^{2}-3 n x_{1}^{*}-3\left(x_{1}^{*}\right)^{2}\right)}{\left(m^{2}+n x_{1}^{*}+\left(x_{1}^{*}\right)^{2}\right)^{3}}, \\
& a_{17}=\frac{a_{1} x_{2}^{*}\left(m^{2}-n^{2}-6 n x_{1}^{*}-9\left(x_{1}^{*}\right)^{2}\right)}{3\left(m^{2}+n x_{1}^{*}+\left(x_{1}^{*}\right)^{2}\right)^{3}} \\
& -\frac{a_{1} x_{1}^{*} x_{2}^{*}\left(m^{2}-n^{2}-3 n x_{1}^{*}-3\left(x_{1}^{*}\right)^{2}\right)\left(n+2 x_{1}^{*}\right)}{\left(m^{2}+n x_{1}^{*}+\left(x_{1}^{*}\right)^{2}\right)^{4}} \\
& c_{23}=-\frac{a_{2}\left(x_{2}^{*}\right)^{2}}{\left(x_{1}^{*}\right)^{3}}, \quad c_{24}=\frac{a_{2} x_{2}^{*}}{\left(x_{1}^{*}\right)^{2}}, \\
& c_{25}=-\frac{a_{2}}{x_{1}^{*}}, \quad c_{26}=\frac{a_{2} x_{2}^{*}}{\left(x_{1}^{*}\right)^{2}}, \\
& c_{27}=\frac{a_{2}\left(x_{2}^{*}\right)^{2}}{\left(x_{1}^{*}\right)^{4}}, \quad c_{28}=-\frac{a_{2} x_{2}^{*}}{\left(x_{1}^{*}\right)^{3}}, \\
& c_{29}=-\frac{a_{2} x_{2}^{*}}{\left(x_{1}^{*}\right)^{3}}, \quad c_{210}=\frac{a_{2}}{\left(x_{1}^{*}\right)^{2}} \text {. }
\end{aligned}
$$


The linearized system of (5) is

$$
\begin{gathered}
\frac{d x_{1}(t)}{d t}=a_{11} x_{1}(t)+a_{12} x_{2}(t)+b_{11} x_{1}\left(t-\tau_{1}\right), \\
\frac{d x_{2}(t)}{d t}=c_{21} x_{1}\left(t-\tau_{2}\right)+c_{22} x_{2}\left(t-\tau_{2}\right) .
\end{gathered}
$$

The associated characteristic equation of system (7) is

$$
\lambda^{2}+A \lambda+B \lambda e^{-\lambda \tau_{1}}+(C \lambda+D) e^{-\lambda \tau_{2}}+E e^{-\lambda\left(\tau_{1}+\tau_{2}\right)}=0,
$$

where

$$
\begin{gathered}
A=-a_{11}, \quad B=-b_{11}, \quad C=-c_{22}, \\
D=a_{11} c_{22}-a_{12} c_{21}, \quad E=b_{11} c_{22} .
\end{gathered}
$$

Case $1\left(\tau_{1}=\tau_{2}=0\right)$. Equation (8) becomes

$$
\lambda^{2}+(A+B+C) \lambda+D+E=0 .
$$

All the roots of (10) have negative real parts if and only if

$$
\left(H_{1}\right) A+B+C>0 \text { and } D+E>0 .
$$

Thus, the positive equilibrium $E^{*}\left(x_{1}^{*}, x_{2}^{*}\right)$ is locally stable when the condition $\left(H_{1}\right)$ holds.

Case $2\left(\tau_{1}>0, \tau_{2}=0\right)$. Equation (8) becomes

$$
\lambda^{2}+(A+C) \lambda+D+(B \lambda+E) e^{-\lambda \tau_{1}}=0 .
$$

Let $\lambda=i \omega_{1}\left(\omega_{1}>0\right)$ be the root of (11), and we have

$$
\begin{gathered}
B \omega_{1} \sin \tau_{1} \omega_{1}+E \cos \tau_{1} \omega_{1}=\omega_{1}^{2}-D, \\
B \omega \cos \tau_{1} \omega_{1}-E \sin \tau_{1} \omega_{1}=-(A+C) \omega_{1} .
\end{gathered}
$$

It follows that

$$
\omega_{1}^{4}+\left((A+C)^{2}-B^{2}-2 D\right) \omega_{1}^{2}+D^{2}-E^{2}=0 .
$$

If condition $\left(\mathrm{H}_{2}\right) D-E<0$ holds, then (13) has only one positive root

$$
\begin{aligned}
\omega_{10}=(( & -\left((A+C)^{2}-B^{2}-2 D\right) \\
& \left.+\sqrt{\left((A+C)^{2}-B^{2}-2 D\right)^{2}-4\left(D^{2}-E^{2}\right)}\right) \\
& \left.\times 2^{-1}\right)^{1 / 2} .
\end{aligned}
$$

Now, we can get the critical value of $\tau_{1}$ by substituting $\omega_{10}$ into (12). After computing, we obtain

$$
\begin{array}{r}
\tau_{1 k}=\frac{1}{\omega_{10}} \arccos \frac{(E-A B-B C) \omega_{10}^{2}-D E}{B^{2} \omega_{10}^{2}+E^{2}}+\frac{2 k \pi}{\omega_{10}}, \\
k=0,1,2 \ldots .
\end{array}
$$

Then, when $\tau=\tau_{1 k}$, the characteristic equation (11) has a pair of purely imaginary roots $\pm i \omega_{0}$.
Next, we will verify the transversality condition. Differentiating (11) with respect to $\tau_{1}$, we get

$$
\left[\frac{d \lambda}{d \tau_{1}}\right]^{-1}=-\frac{2 \lambda+A+C}{\lambda\left[\lambda^{2}+(A+C) \lambda+D\right]}+\frac{B}{\lambda(B \lambda+E)}-\frac{\tau_{1}}{\lambda} .
$$

Thus,

$$
\begin{aligned}
\operatorname{Re}\left[\frac{d \lambda}{d \tau_{1}}\right]_{\tau_{1}=\tau_{10}}^{-1} & =\frac{2 \omega_{10}^{2}+(A+C)^{2}-B^{2}-2 D}{B^{2} \omega_{10}^{2}+E^{2}} \\
& =\frac{\sqrt{\left((A+C)^{2}-B^{2}-2 D\right)^{2}-4\left(D^{2}-E^{2}\right)}}{B^{2} \omega_{10}^{2}+E^{2}} .
\end{aligned}
$$

Obviously, if condition $\left(H_{2}\right)$ holds, then $\operatorname{Re}\left[d \lambda / d \tau_{1}\right]_{\tau_{1}=\tau_{0}}^{-1}>0$. Note that $\operatorname{sgn}\left\{\operatorname{Re}\left[d \lambda / d \tau_{1}\right]_{\tau_{1}=\tau_{10}}^{-1}\right\}=\operatorname{sgn}\left\{\left[d \operatorname{Re}(\lambda) / d \tau_{1}\right]_{\tau_{1}=\tau_{10}}^{-1}\right\}$.

Therefore, if condition $\left(\mathrm{H}_{2}\right)$ holds, the transversality condition is satisfied. From the analysis above, we have the following results.

Theorem 1. If conditions $\left(H_{1}\right)$ and $\left(H_{2}\right)$ hold, then the positive equilibrium $E^{*}\left(x_{1}^{*}, x_{2}^{*}\right)$ of system (3) is asymptotically stable for $\tau_{1} \in\left[0, \tau_{10}\right)$ and unstable when $\tau_{1}>\tau_{10}$. System (3) undergoes a Hopf bifurcation at the positive equilibrium $E^{*}\left(x_{1}^{*}, x_{2}^{*}\right)$ when $\tau_{1}=\tau_{10}$.

Case $3\left(\tau_{1}=0, \tau_{2}>0\right)$. Equation (8) becomes

$$
\lambda^{2}+(A+B) \lambda+(C \lambda+D+E) e^{-\lambda \tau_{2}}=0 .
$$

Let $\lambda=i \omega_{2}\left(\omega_{2}>0\right)$ be the root of (18), and then we have

$$
\begin{gathered}
C \omega_{2} \sin \tau_{2} \omega_{2}+(D+E) \cos \tau_{2} \omega_{2}=\omega_{2}^{2}, \\
C \omega_{2} \cos \tau_{2} \omega_{2}-(D+E) \sin \tau_{2} \omega_{2}=-(A+B) \omega_{2},
\end{gathered}
$$

which leads to

$$
\omega_{2}^{4}+\left((A+B)^{2}-C^{2}\right) \omega_{2}^{2}-(D+E)^{2}=0 .
$$

Obviously, if the condition $\left(H_{1}\right)$ holds, and then (20) has only one positive root

$$
=\sqrt{\frac{-\left((A+B)^{2}-C^{2}\right)+\sqrt{\left((A+B)^{2}-C^{2}\right)^{2}+4(D+E)^{2}}}{2}} .
$$

The corresponding critical value of $\tau_{2}$ is

$$
\begin{array}{r}
\tau_{2 k}=\frac{1}{\omega_{20}} \arccos \frac{(D+E-A C-B C) \omega_{10}^{2}}{C^{2} \omega_{20}^{2}+(D+E)^{2}}+\frac{2 k \pi}{\omega_{20}}, \\
k=0,1,2, \ldots .
\end{array}
$$


Similar to Case 2, differentiating (18) with respect to $\tau_{2}$, we get

$$
\left[\frac{d \lambda}{d \tau_{2}}\right]^{-1}=-\frac{2 \lambda+A+B}{\lambda\left[\lambda^{2}+(A+B) \lambda\right]}+\frac{C}{\lambda(C \lambda+D+E)}-\frac{\tau_{2}}{\lambda} .
$$

Then we can get

$$
\begin{aligned}
\operatorname{Re}\left[\frac{d \lambda}{d \tau_{2}}\right]_{\tau_{2}=\tau_{20}}^{-1} & =\frac{2 \omega_{20}^{2}+(A+B)^{2}-C^{2}}{C^{2} \omega_{20}^{2}+(D+E)^{2}} \\
& =\frac{\sqrt{\left((A+B)^{2}-C^{2}\right)^{2}-4(D+E)^{2}}}{C^{2} \omega_{20}^{2}+(D+E)^{2}} .
\end{aligned}
$$

Obviously, if condition $\left(H_{1}\right)$ holds, then $\operatorname{Re}[d \lambda /$ $\left.d \tau_{2}\right]_{\tau_{2}=\tau_{20}}^{-1}>0$. Namely, if condition $\left(H_{1}\right)$ holds, then the transversality condition is satisfied. Thus, we have the following results.

Theorem 2. If condition $\left(H_{1}\right)$ holds, then the positive equilibrium $E^{*}\left(x_{1}^{*}, x_{2}^{*}\right)$ of system (3) is asymptotically stable for $\tau_{2} \in\left[0, \tau_{20}\right)$ and unstable when $\tau_{2}>\tau_{20}$. System (3) undergoes a Hopf bifurcation at the positive equilibrium $E^{*}\left(x_{1}^{*}, x_{2}^{*}\right)$ when $\tau_{2}=\tau_{20}$.

Case $4\left(\tau_{1}=\tau_{2}=\tau>0\right)$. Equation (8) can be transformed into the following form:

$$
\lambda^{2}+A \lambda+((B+C) \lambda+D) e^{-\lambda \tau}+E e^{-2 \lambda \tau}=0 .
$$

Multiplying $e^{\lambda \tau}$ on both sides of (25), we get

$$
(B+C) \lambda+D+\left(\lambda^{2}+A \lambda\right) e^{\lambda \tau}+E e^{-\lambda \tau}=0 .
$$

Let $\lambda=i \omega(\omega>0)$ be the root of $(26)$, then we obtain

$$
\begin{gathered}
A \omega \sin \tau \omega+\left(\omega^{2}-E\right) \cos \tau \omega=D, \\
A \omega \cos \tau \omega-\left(\omega^{2}+E\right) \sin \tau \omega=-(B+C) \omega,
\end{gathered}
$$

which follows that

$$
\begin{gathered}
\sin \tau \omega=\frac{(B+C) \omega^{3}+(A D-B E-C E) \omega}{\omega^{4}+A^{2} \omega^{2}-E^{2}}, \\
\cos \tau \omega=\frac{(D-A B-A C) \omega^{2}+D E}{\omega^{4}+A^{2} \omega^{2}-E^{2}} .
\end{gathered}
$$

Then, we have

$$
\omega^{8}+c_{3} \omega^{6}+c_{2} \omega^{4}+c_{1} \omega^{2}+c_{0}=0
$$

where

$$
\begin{aligned}
c_{0}= & E^{4}, \quad c_{3}=2 A^{2}-(B+C)^{2}, \\
c_{1}= & 2 D E(A B+A C-D)-2 A^{2} E^{2} \\
& -(A D-B E-C E)^{2}, \\
c_{2}= & A^{4}-2 E^{2}-2(B+C)(A D-B E-C E) \\
& -(D-A B-A C)^{2} .
\end{aligned}
$$

Denote $\omega^{2}=v$, and then (29) becomes

$$
v^{4}+c_{3} v^{3}+c_{2} v^{2}+c_{1} v+c_{0}=0 .
$$

Next, we give the following assumption.

$\left(\mathrm{H}_{3}\right)$ Equation (31) has at least one positive real root.

Suppose that $\left(\mathrm{H}_{3}\right)$ holds. Without loss of generality, we assume that (31) has four real positive roots, which are denoted by $v_{1}, v_{2}, v_{3}$, and $v_{4}$. Then (29) has four positive roots $\omega_{k}=\sqrt{v_{k}}, k=1,2,3,4$.

For every fixed $\omega_{k}$, the corresponding critical value of time delay is

$$
\begin{array}{r}
\tau_{k}^{(j)}=\frac{1}{\omega_{k}} \arccos \frac{(D-A B-A C) \omega_{k}^{2}+D E}{\omega_{k}^{4}+A^{2} \omega_{k}^{2}-E^{2}}+\frac{2 j \pi}{\omega_{k}}, \\
k=1,2,3,4, j=0,1,2, \ldots
\end{array}
$$

Let

$$
\tau_{0}=\min \left\{\tau_{k}^{(0)}\right\}, \quad k=1,2,3,4 . \quad \omega_{0}=\omega_{k_{0}} .
$$

Taking the derivative of $\lambda$ with respect to $\tau$ in (26), we obtain

$$
\left[\frac{d \lambda}{d \tau}\right]^{-1}=\frac{(2 \lambda+A) e^{\lambda \tau}+B+C}{\lambda\left[E e^{-\lambda \tau}-\left(\lambda^{2}+A \lambda\right) e^{\lambda \tau}\right]}-\frac{\tau}{\lambda} .
$$

Thus,

$$
\operatorname{Re}\left[\frac{d \lambda}{d \tau}\right]_{\tau=\tau_{0}}^{-1}=\frac{P_{R} Q_{R}+P_{I} Q_{I}}{Q_{R}^{2}+Q_{I}^{2}}
$$

with

$$
\begin{gathered}
P_{R}=A \cos \tau_{0} \omega_{0}-2 \omega_{0} \sin \tau_{0} \omega_{0}+B+C, \\
P_{I}=A \sin \tau_{0} \omega_{0}+2 \omega_{0} \cos \tau_{0} \omega_{0}, \\
Q_{R}=A \omega_{0}^{2} \cos \tau_{0} \omega_{0}+\left(E \omega_{0}-\omega_{0}^{3}\right) \sin \tau_{0} \omega_{0}, \\
Q_{I}=A \omega_{0}^{2} \sin \tau_{0} \omega_{0}+\left(E \omega_{0}+\omega_{0}^{3}\right) \cos \tau_{0} \omega_{0} .
\end{gathered}
$$

It is easy to see that if condition $\left(H_{4}\right) P_{R} Q_{R}+P_{I} Q_{I} \neq 0$, then $\operatorname{Re}[d \lambda / d \tau]_{\tau=\tau_{0}}^{-1} \neq 0$. Therefore, we have the following results.

Theorem 3. If conditions $\left(H_{1}\right)$ and $\left(H_{4}\right)$ holds, then the positive equilibrium $E^{*}\left(x_{1}^{*}, x_{2}^{*}\right)$ of system (3) is asymptotically stable for $\tau \in\left[0, \tau_{0}\right)$ and unstable when $\tau>\tau_{0}$. System (3) undergoes a Hopf bifurcation at the positive equilibrium $E^{*}\left(x_{1}^{*}, x_{2}^{*}\right)$ when $\tau=\tau_{0}$.

Case $5\left(\tau_{1} \neq \tau_{2}, \tau_{1}>0\right.$ and $\left.\tau_{2}>0\right)$. Considered the following:

$$
\begin{aligned}
& \lambda^{2}+A \lambda+B \lambda e^{-\lambda \tau_{1}}+(C \lambda+D) e^{-\lambda \tau_{2}} \\
& +E e^{-\lambda\left(\tau_{1}+\tau_{2}\right)}=0 .
\end{aligned}
$$

We consider (8) with $\tau_{2}$ in its stable interval and regard $\tau_{1}$ as a parameter. 
Let $\lambda=i \omega_{1}^{*}\left(\omega_{1}^{*}>0\right)$ be the root of $(8)$, and then we can obtain

$$
\begin{gathered}
\left(B \omega_{1}^{*}-E \sin \tau_{2} \omega_{1}^{*}\right) \sin \tau_{1} \omega_{1}^{*}+E \cos \tau_{2} \omega_{1}^{*} \cos \tau_{1} \omega_{1}^{*} \\
=\left(\omega_{1}^{*}\right)^{2}-D \cos \tau_{2} \omega_{1}^{*}-C \omega_{1}^{*} \sin \tau_{2} \omega_{1}^{*}, \\
\left(B \omega_{1}^{*}-E \sin \tau_{2} \omega_{1}^{*}\right) \cos \tau_{1} \omega_{1}^{*}-E \cos \tau_{2} \omega_{1}^{*} \cos \tau_{1} \omega_{1}^{*} \\
=-A \omega_{1}^{*}+D \sin \tau_{2} \omega_{1}^{*}-C \omega_{1}^{*} \cos \tau_{2} \omega_{1}^{*} .
\end{gathered}
$$

Then we get

$$
\begin{aligned}
& \left(\omega_{1}^{*}\right)^{4}+\left(A^{2}-B^{2}+C^{2}\right)\left(\omega_{1}^{*}\right)^{2}+D^{2}-E^{2} \\
& \quad+2\left(A C-D^{2}\right)\left(\omega_{1}^{*}\right)^{2} \cos \tau_{2} \omega_{1}^{*} \\
& \quad-2\left(C\left(\omega_{1}^{*}\right)^{3}+A D \omega_{1}^{*}\right) \sin \tau_{2} \omega_{1}^{*}=0 .
\end{aligned}
$$

Define

$$
\begin{aligned}
F\left(\omega_{1}^{*}\right)= & \left(\omega_{1}^{*}\right)^{4}+\left(A^{2}-B^{2}+C^{2}\right)\left(\omega_{1}^{*}\right)^{2}+D^{2}-E^{2} \\
& +2\left(A C-D^{2}\right)\left(\omega_{1}^{*}\right)^{2} \cos \tau_{2} \omega_{1}^{*} \\
& -2\left(C\left(\omega_{1}^{*}\right)^{3}+A D \omega_{1}^{*}\right) \sin \tau_{2} \omega_{1}^{*} .
\end{aligned}
$$

If condition $\left(H_{1}\right) D+E>0$ and $\left(H_{2}\right) D-E<0$ hold, then $F(0)=D^{2}-E^{2}<0$. In addition, $F(+\infty)=+\infty$. Therefore, (39) has at least one positive root. We suppose that the positive roots of (39) are denoted as $\omega_{11}^{*}, \omega_{12}^{*}, \ldots \omega_{1 k}^{*}$. For every fixed $\omega_{1 i}^{*}$, the corresponding critical value of time delay

$$
\begin{aligned}
\tau_{1 i}^{*(j)}= & \frac{1}{\omega_{1 i}^{*}} \\
& \times \arccos \frac{e_{1}\left(\omega_{1 i}^{*}\right)+e_{2}\left(\omega_{1 i}^{*}\right) \cos \tau_{2} \omega_{1 i}^{*}+e_{3}\left(\omega_{1 i}^{*}\right) \sin \tau_{2} \omega_{1 i}^{*}}{e_{4}\left(\omega_{1 i}^{*}\right)+e_{5}\left(\omega_{1 i}^{*}\right) \sin \tau_{2} \omega_{1 i}^{*}} \\
+ & \frac{2 j \pi}{\omega_{1 i}^{*}} \quad i=1,2,3, \ldots, k, j=0,1,2, \ldots
\end{aligned}
$$

with

$$
\begin{gathered}
e_{1}\left(\omega_{1 i}^{*}\right)=-A B\left(\omega_{1 i}^{*}\right)^{2}-D E, \quad e_{2}\left(\omega_{1 i}^{*}\right)=(E-B C)\left(\omega_{1 i}^{*}\right)^{2}, \\
e_{3}\left(\omega_{1 i}^{*}\right)=(A E+B D) \omega_{1 i}^{*}, \quad e_{4}\left(\omega_{1 i}^{*}\right)=B^{2}\left(\omega_{1 i}^{*}\right)^{2}+E^{2}, \\
e_{5}\left(\omega_{1 i}^{*}\right)=-2 B E \omega_{1 i}^{*} .
\end{gathered}
$$

Let $\tau_{1 *}=\min \left\{\tau_{1 i}^{*(0)}\right\}, i=1,2,3, \ldots k$. When $\tau_{1}=\tau_{1 *}$, (8) has a pair of purely imaginary roots $\pm i \omega_{1 *}$ for $\tau_{2} \in\left(0, \tau_{20}\right)$.

Next, in order to give the main results, we give the following assumption

$\left(H_{5}\right) d \operatorname{Re} \lambda\left(\tau_{10}^{*}\right) / d \tau_{1} \neq 0$.

Therefore, we have the following results on the stability and bifurcation in system (3).
Theorem 4. If the conditions $\left(H_{1}\right)$ and $\left(H_{5}\right)$ hold and $\tau_{2} \epsilon$ $\left(0, \tau_{20}\right)$, then the positive equilibrium $E^{*}\left(x_{1}^{*}, x_{2}^{*}\right)$ of system (3) is asymptotically stable for $\tau_{1} \in\left[0, \tau_{1 *}\right)$ and unstable when $\tau_{1}>\tau_{1 *}$. System (3) undergoes a Hopf bifurcation at the positive equilibrium $E^{*}\left(x_{1}^{*}, x_{2}^{*}\right)$ when $\tau_{1}=\tau_{1 *}$.

\section{Direction and Stability of the Hopf Bifurcation}

In this section, we will investigate the direction of Hopf bifurcation and stability of the bifurcating periodic solutions of system (3) with respect to $\tau_{1}$ for $\tau_{2} \in\left(0, \tau_{20}\right)$. We assume that $\tau_{2 *}<\tau_{1 *}$ where $\tau_{2 *} \in\left(0, \tau_{20}\right)$.

Let $\tau_{1}=\tau_{1 *}+\mu, \mu \in R$ so that $\mu=0$ is the Hopf bifurcation value of system (3). Rescale the time delay by $t \rightarrow\left(t / \tau_{1}\right)$. Let $u_{1}(t)=x_{1}(t)-x_{1}^{*}, u_{2}(t)=x_{2}(t)-x_{2}^{*}$, and then system (3) can be rewritten as an PDE in $C=C\left([-1,0], R^{2}\right)$ as

$$
\dot{u}(t)=L_{\mu} u_{t}+F\left(\mu, u_{t}\right) \text {, }
$$

where

$$
\begin{gathered}
u(t)=\left(u_{1}(t), u_{2}(t)\right)^{T} \in R^{2}, \\
L_{\mu}: C \longrightarrow R^{2}, \quad F: R \times C \longrightarrow R^{2}
\end{gathered}
$$

are given, respectively, by

$$
\begin{gathered}
L_{\mu} \phi=\left(\tau_{1 *}+\mu\right)\left(A^{\prime} \phi(0)+C^{\prime} \phi\left(-\frac{\tau_{2 *}}{\tau_{1 *}}\right)+B^{\prime} \phi(-1)\right), \\
F\left(\mu, u_{t}\right)=\left(\tau_{1 *}+\mu\right)\left(F_{1}, F_{2}\right)^{T}
\end{gathered}
$$

with

$$
\begin{gathered}
\phi(\theta)=\left(\phi_{1}(\theta), \phi_{2}(\theta)\right)^{T} \in C\left([-1,0], R^{2}\right), \\
A^{\prime}=\left(\begin{array}{cc}
a_{11} & a_{12} \\
0 & 0
\end{array}\right), \quad B^{\prime}=\left(\begin{array}{cc}
b_{11} & 0 \\
0 & 0
\end{array}\right), \\
C^{\prime}=\left(\begin{array}{cc}
0 & 0 \\
c_{21} & c_{22}
\end{array}\right), \\
F_{1}=a_{13} \phi_{1}^{2}(0)+a_{14} \phi_{1}(0) \phi_{2}(0)+a_{15} \phi_{1}(0) \phi_{1}(-1) \\
+a_{16} \phi_{1}^{2}(0) \phi_{2}(0)+a_{17} \phi_{1}^{3}(0)+\cdots \\
F_{2}=c_{23} \phi_{1}^{2}\left(-\frac{\tau_{2 *}}{\tau_{1 *}}\right)+c_{24} \phi_{1}\left(-\frac{\tau_{2 *}}{\tau_{1 *}}\right) \phi_{2}(0) \\
+c_{25} \phi_{2}\left(-\frac{\tau_{2 *}}{\tau_{1 *}}\right) \phi_{2}(0)+c_{26} \phi_{1}\left(-\frac{\tau_{2 *}}{\tau_{1 *}}\right) \phi_{2}\left(-\frac{\tau_{2 *}}{\tau_{1 *}}\right) \\
+c_{27} \phi_{1}^{3}\left(-\frac{\tau_{2 *}}{\tau_{1 *}}\right)+c_{28} \phi_{1}^{2}\left(-\frac{\tau_{2 *}}{\tau_{1 *}}\right) \phi_{2}(0) \\
+c_{29} \phi_{1}^{2}\left(-\frac{\tau_{2 *}}{\tau_{1 *}}\right) \phi_{2}\left(-\frac{\tau_{2 *}}{\tau_{1 *}}\right) \\
+c_{210} \phi_{1}\left(-\frac{\tau_{2 *}}{\tau_{1 *}}\right) \phi_{2}\left(-\frac{\tau_{2 *}}{\tau_{1 *}}\right) \phi_{2}(0)+\cdots .
\end{gathered}
$$


By the Riesz representation theorem, there is a $2 \times 2$ matrix function with bounded variation components $\eta(\theta, \mu), \theta \in$ $[-1,0]$ such that

$$
L_{\mu} \phi=\int_{-1}^{0} d \eta(\theta, \mu) \phi(\theta), \quad \phi \in C .
$$

In fact, we have chosen

$$
\eta(\theta, \mu)= \begin{cases}\left(\tau_{1 *}+\mu\right)\left(A^{\prime}+B^{\prime}+C^{\prime}\right), & \theta=0, \\ \left(\tau_{1 *}+\mu\right)\left(B^{\prime}+C^{\prime}\right), & \theta \in\left[-\frac{\tau_{2 *}}{\tau_{1 *}}, 0\right), \\ \left(\tau_{1 *}+\mu\right) B^{\prime}, & \theta \in\left(-1,-\frac{\tau_{2 *}}{\tau_{1 *}}\right), \\ 0, & \theta=-1 .\end{cases}
$$

For $\phi \in C([-1,0]), R^{2}$, we define

$$
\begin{gathered}
A(\mu) \phi= \begin{cases}\frac{d \phi(\theta)}{d \theta}, & -1 \leq \theta<0, \\
\int_{-1}^{0} d \eta(\theta, \mu) \phi(\theta), & \theta=0,\end{cases} \\
R(\mu) \phi= \begin{cases}0, & -1 \leq \theta<0, \\
F(\mu, \phi), & \theta=0 .\end{cases}
\end{gathered}
$$

Then system (43) is equivalent to the following operator equation:

$$
\dot{u}(t)=A(\mu) u_{t}+R(\mu) u_{t},
$$

where $u_{t}=u(t+\theta)$ for $\theta \in[-1,0]$.

For $\varphi \in C([-1,0]),\left(R^{2}\right)^{*}$, we define the adjoint operator $A^{*}$ of $A$ as

$$
A^{*}(\mu) s= \begin{cases}-\frac{d \varphi(s)}{d s}, & 0<s \leq 1, \\ \int_{-1}^{0} d \eta^{T}(s, \mu) \varphi(-s), & s=0,\end{cases}
$$

and a bilinear inner product

$$
\langle\varphi, \phi\rangle=\bar{\varphi}(0) \phi(0)-\int_{\theta=-1}^{0} \int_{\xi=0}^{\theta} \bar{\varphi}(\xi-\theta) d \eta(\theta) \phi(\xi) d \xi,
$$

where $\eta(\theta)=\eta(\theta, 0)$.

Then, $A(0)$ and $A^{*}(0)$ are adjoint operators. From the discussion above, we can know that $\pm i \omega_{1 *} \tau_{1 *}$ are the eigenvalues of $A(0)$ and they are also eigenvalues of $A^{*}(0)$.

Let $q(\theta)=\left(1, q_{2}\right)^{T} e^{i \omega_{1 *} \tau_{1 *} \theta}$ be the eigenvector of $A(0)$ corresponding to $i \omega_{1 *} \tau_{1 *}$, and $q^{*}(\theta)=D\left(1, q_{2}^{*}\right) e^{i \omega_{1 *} \tau_{1 *} s}$ be the eigenvector of $A^{*}(0)$ corresponding to $-i \omega_{1 *} \tau_{1 *}$. Then we have

$$
\begin{gathered}
A(0) q(\theta)=i \omega_{1 *} \tau_{1 *} q(\theta), \\
A^{*}(0) q^{*}(\theta)=-i \omega_{1 *} \tau_{1 *} q^{*}(\theta) .
\end{gathered}
$$

By a simple computation, we can get

$$
\begin{aligned}
& q_{2}=\frac{i \omega_{1 *}-a_{11}-b_{11} e^{-i \omega_{1 *} \tau_{1 *}}}{a_{12}}, \\
& q_{2}^{*}=\frac{i \omega_{1 *}-a_{11}-b_{11} e^{i \omega_{1 *} \tau_{1 *}}}{c_{21} e^{i \omega_{1 *} \tau_{2 *}}}
\end{aligned}
$$

$$
\begin{gathered}
\bar{D}=\left[1+q_{2} \bar{q}_{2}^{*}+b_{11} \tau_{1 *} e^{-i \omega_{1 *} \tau_{1 *}}\right. \\
\left.+\bar{q}_{2}^{*}\left(c_{21}+c_{22} q_{2}\right) \tau_{2 *} e^{-i \omega_{1 *} \tau_{2 *}}\right]^{-1} . \\
\left\langle q^{*}, q\right\rangle=1, \quad\left\langle q^{*}, \bar{q}\right\rangle=0 .
\end{gathered}
$$

Then,

Following the algorithms explained in the work of Hassard et al. [22] and using a computation process similar to that in [18], we can get the following coefficients which can determine the properties of Hopf bifurcation:

$$
\begin{aligned}
& g_{20}=2 \tau_{1 *} \bar{D}\left[a_{13}+a_{14} q^{(2)}(0)+a_{15} q^{(1)}(-1)\right. \\
& +\bar{q}_{2}^{*}\left(c_{23}\left(q^{(1)}\left(-\frac{\tau_{2 *}}{\tau_{1 *}}\right)\right)^{2}+c_{24} q^{(1)}\left(-\frac{\tau_{2 *}}{\tau_{1 *}}\right)\right. \\
& \times q^{(2)}(0)+c_{25} q^{(2)}\left(-\frac{\tau_{2 *}}{\tau_{1 *}}\right) q^{(2)}(0) \\
& \left.\left.+c_{26} q^{(1)}\left(-\frac{\tau_{2 *}}{\tau_{1 *}}\right) q^{(2)}\left(-\frac{\tau_{2 *}}{\tau_{1 *}}\right)\right)\right] \text {, } \\
& g_{11}=\tau_{1 *} \bar{D}\left[2 a_{13}+a_{14}\left(q^{(2)}(0)+\bar{q}^{(2)}(0)\right)\right. \\
& +a_{15}\left(q^{(1)}(-1)+\bar{q}^{(1)}(-1)\right) \\
& +\bar{q}_{2}^{*}\left(2 c_{23} q^{(1)}\left(-\frac{\tau_{2 *}}{\tau_{1 *}}\right) \bar{q}^{(1)}\left(-\frac{\tau_{2 *}}{\tau_{1 *}}\right)\right. \\
& +c_{24}\left(q^{(1)}\left(-\frac{\tau_{2 *}}{\tau_{1 *}}\right) \bar{q}^{(2)}(0)\right. \\
& \left.+\bar{q}^{(1)}\left(-\frac{\tau_{2 *}}{\tau_{1 *}}\right) q^{(2)}(0)\right) \\
& +c_{25}\left(q^{(2)}\left(-\frac{\tau_{2 *}}{\tau_{1 *}}\right) \bar{q}^{(2)}(0)\right. \\
& \left.+\bar{q}^{(2)}\left(-\frac{\tau_{2 *}}{\tau_{1 *}}\right) q^{(2)}(0)\right) \\
& +c_{26}\left(q^{(1)}\left(-\frac{\tau_{2 *}}{\tau_{1 *}}\right) \bar{q}^{(2)}\left(-\frac{\tau_{2 *}}{\tau_{1 *}}\right)\right. \\
& \left.\left.\left.+\bar{q}^{(1)}\left(-\frac{\tau_{2 *}}{\tau_{1 *}}\right) q^{(2)}\left(-\frac{\tau_{2 *}}{\tau_{1 *}}\right)\right)\right)\right],
\end{aligned}
$$




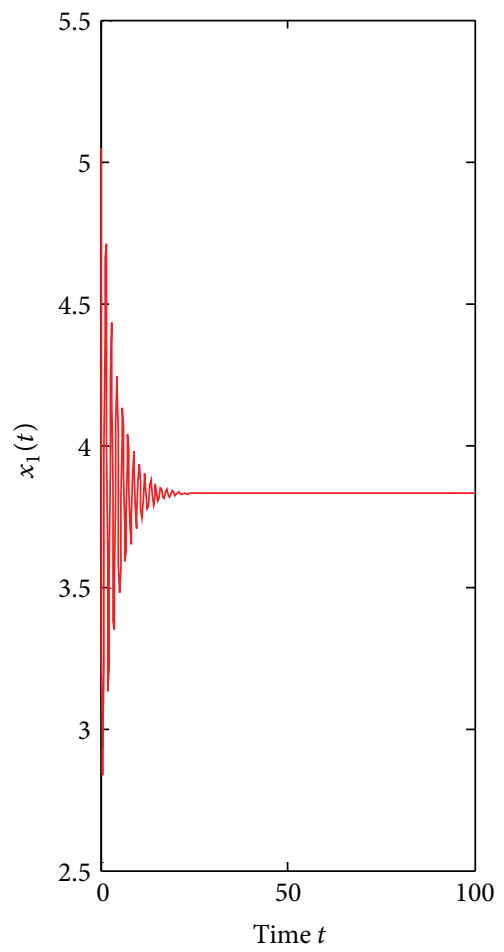

(a)

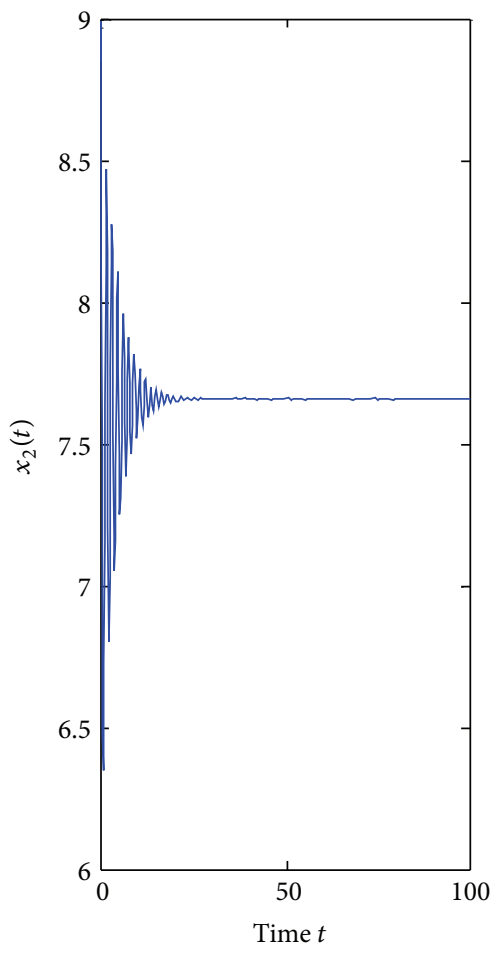

(b)

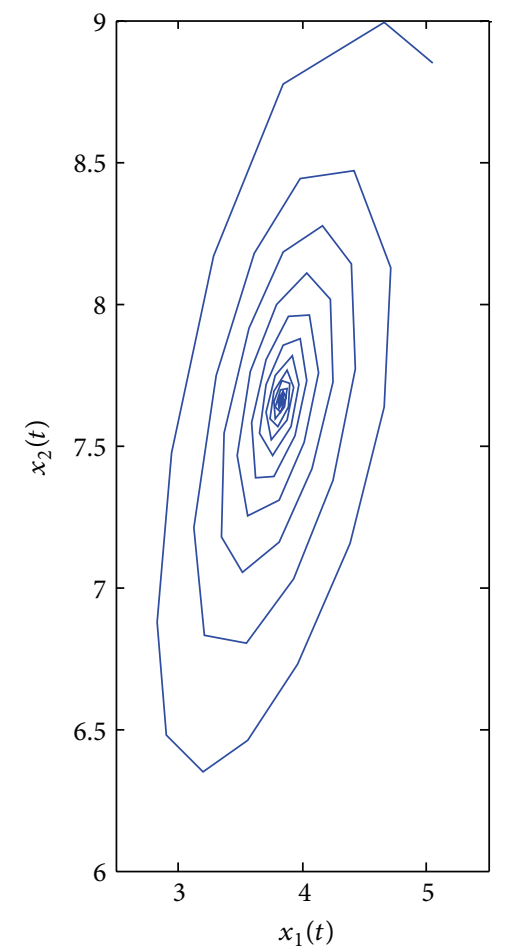

(c)

Figure 1: $E^{*}$ is asymptotically stable for $\tau_{1}=0.350<\tau_{10}=0.3788$.

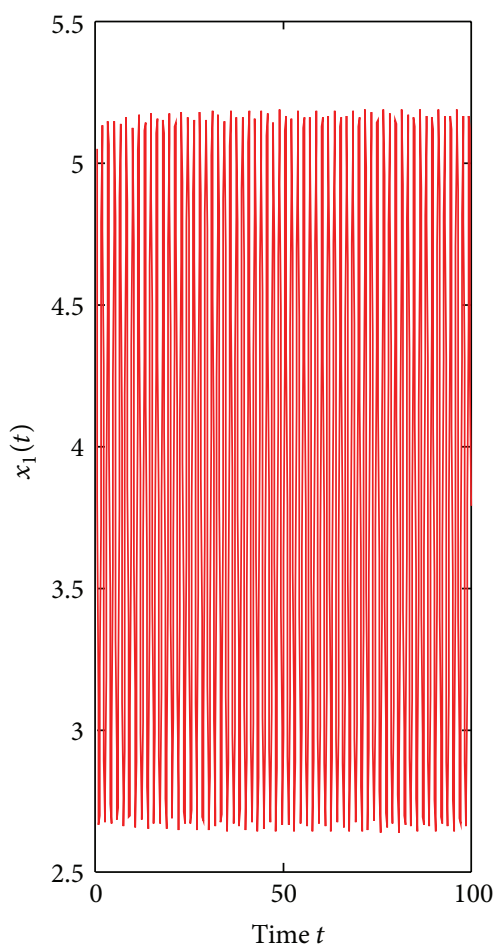

(a)

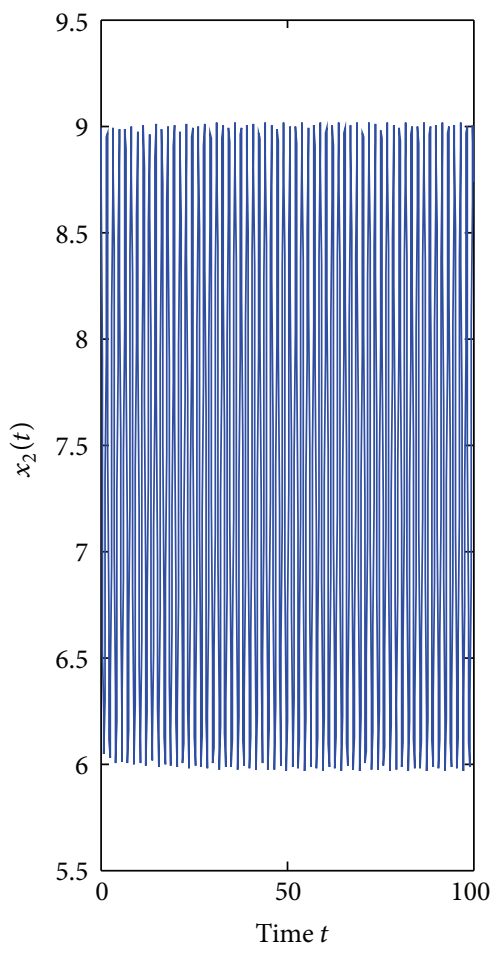

(b)

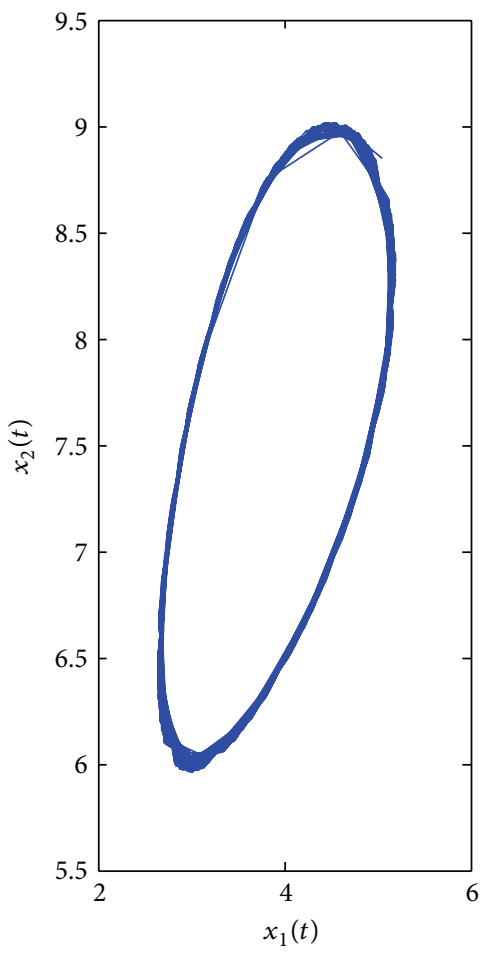

(c)

FIGURE 2: $E^{*}$ is unstable for $\tau_{1}=0.395>\tau_{10}=0.3788$. 


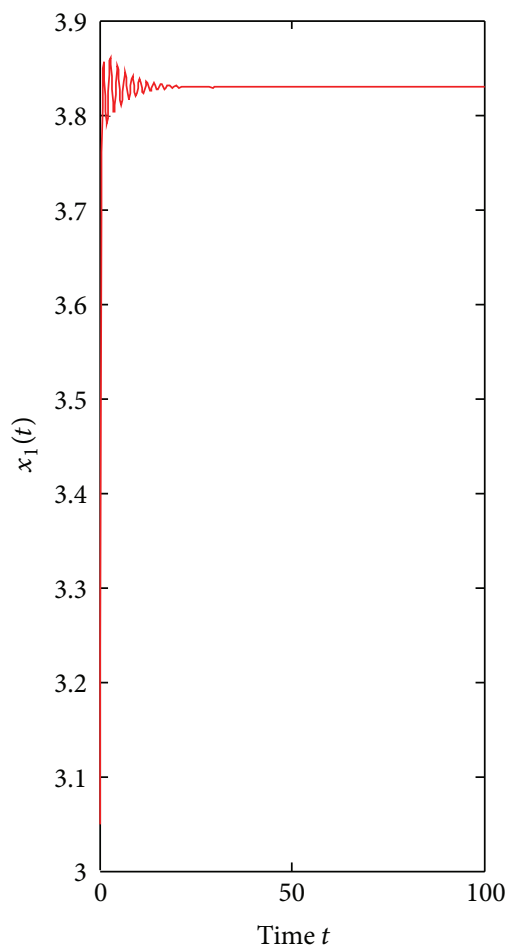

(a)

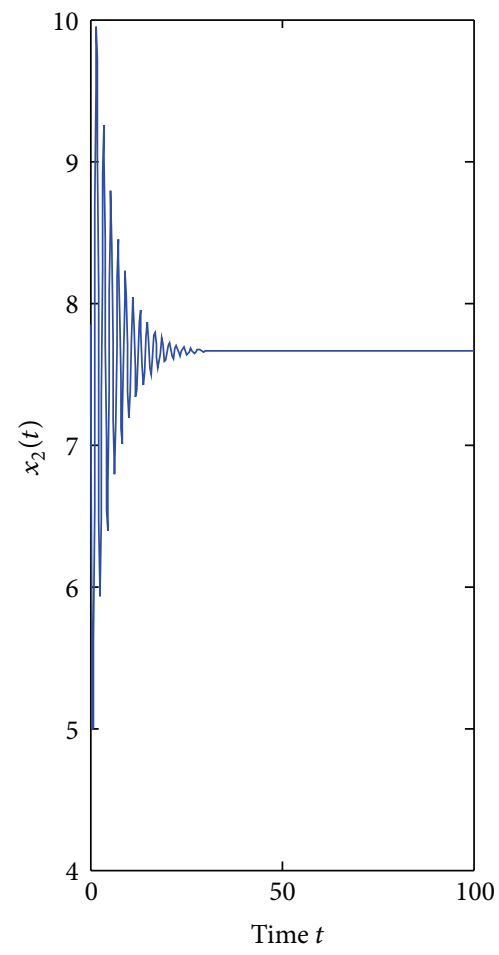

(b)

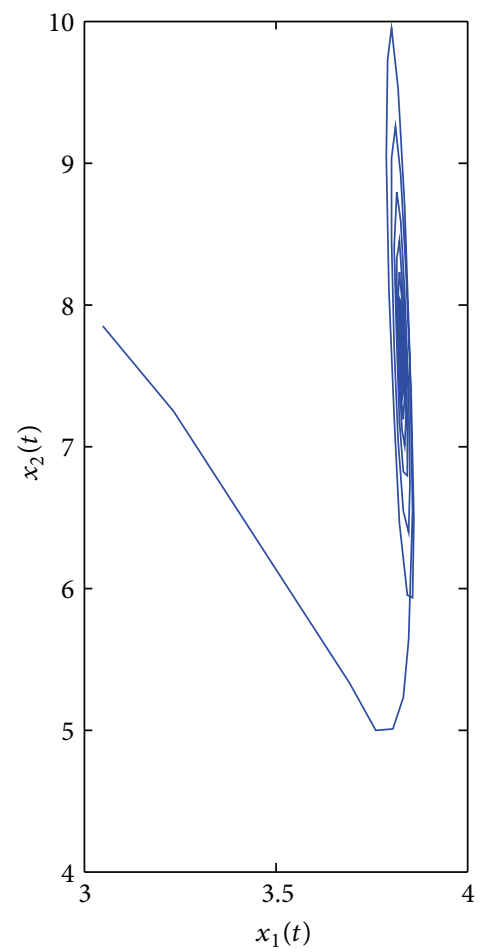

(c)

Figure 3: $E^{*}$ is asymptotically stable for $\tau_{2}=0.450<\tau_{20}=0.4912$.

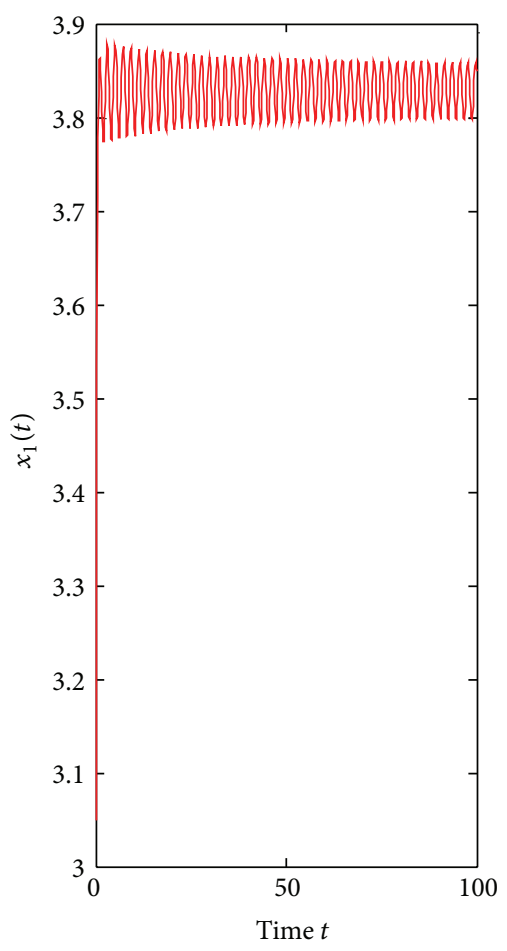

(a)

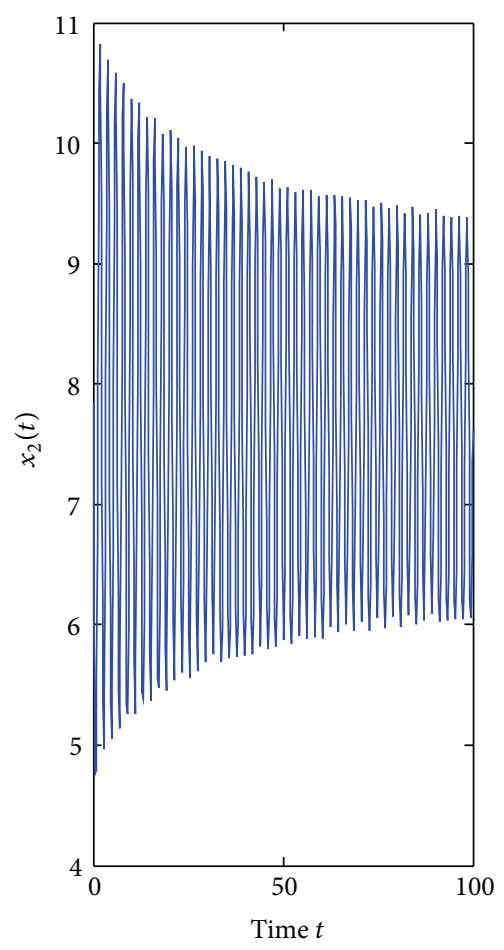

(b)

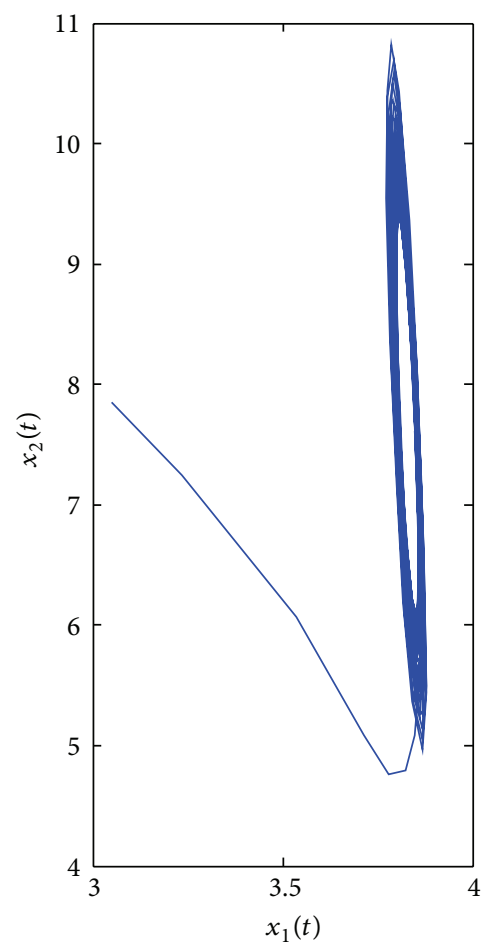

(c)

FIGURE 4: $E^{*}$ is unstable for $\tau_{2}=0.505>\tau_{20}=0.4912$. 


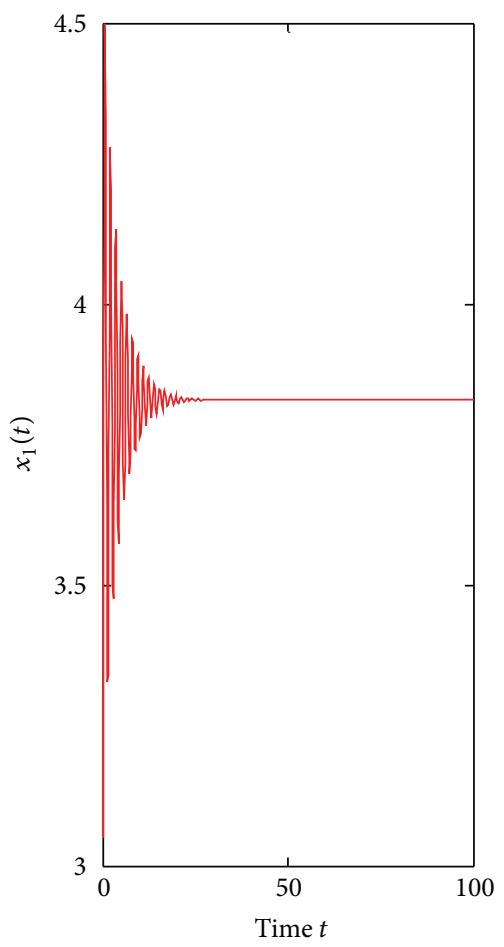

(a)

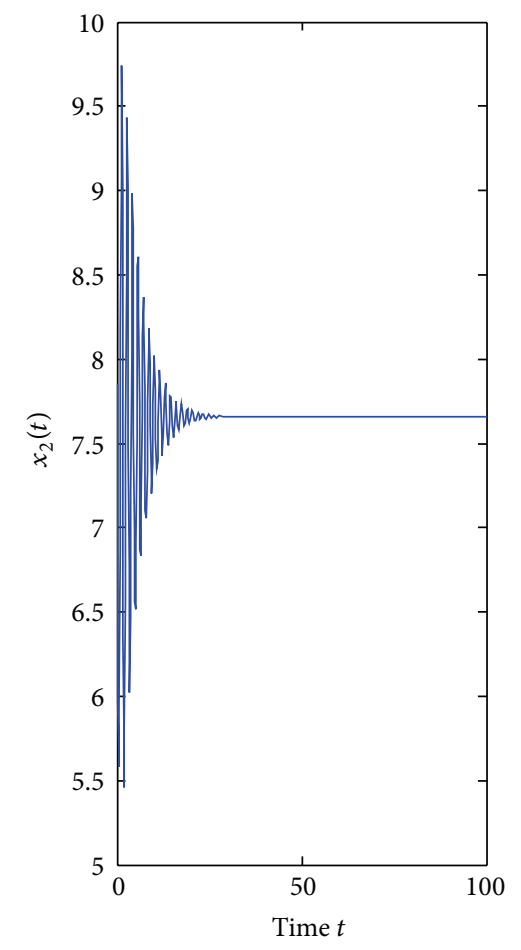

(b)

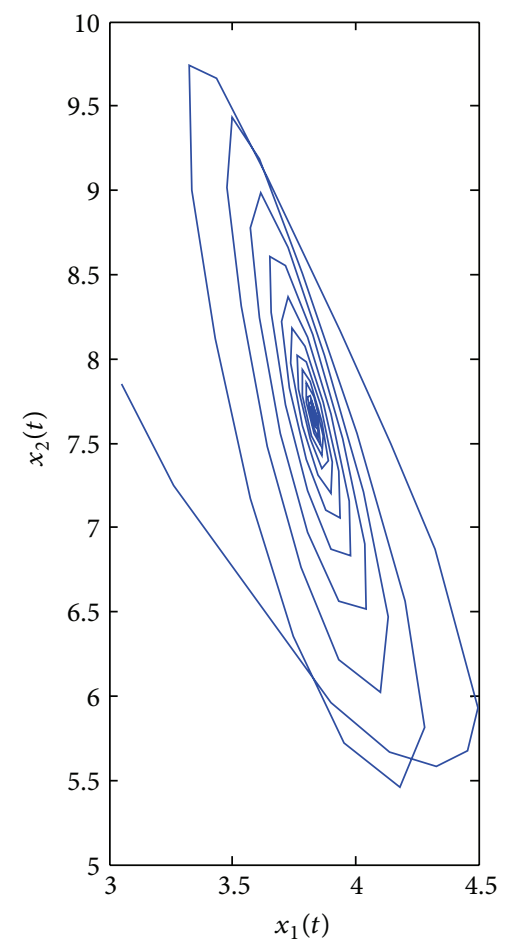

(c)

FIGURE 5: $E^{*}$ is asymptotically stable for $\tau=0.320<\tau_{0}=0.3361$.

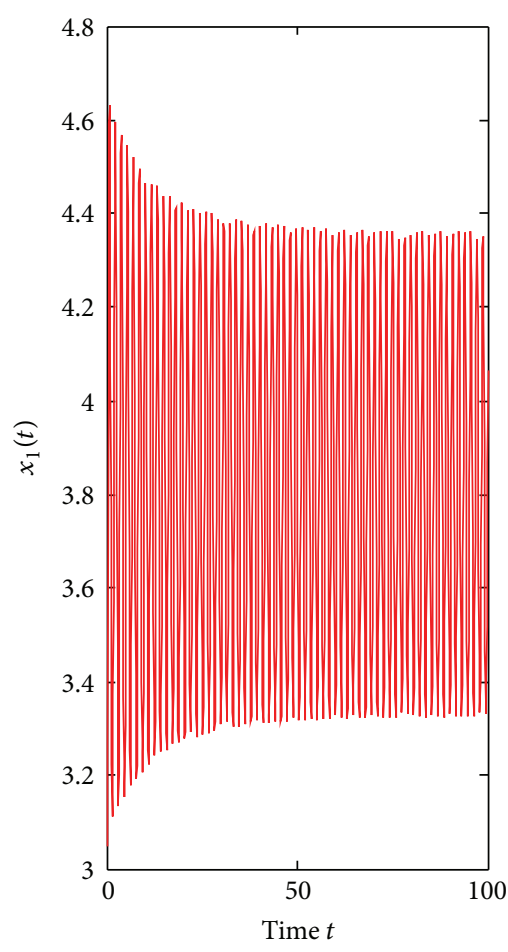

(a)

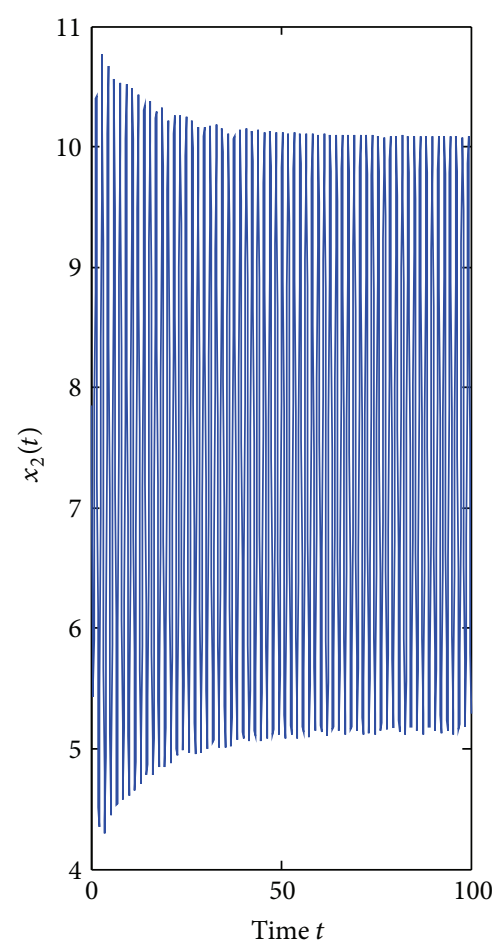

(b)

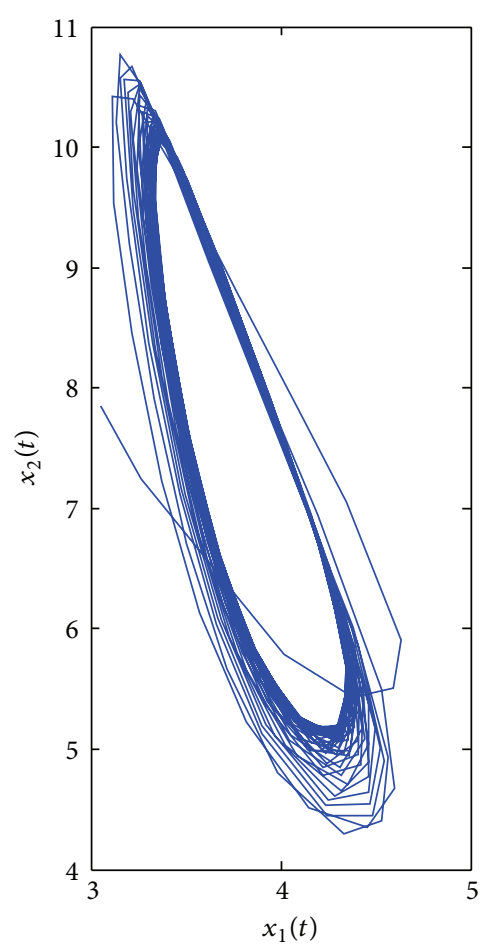

(c)

Figure 6: $E^{*}$ is unstable for $\tau=0.355>\tau_{0}=0.3361$. 


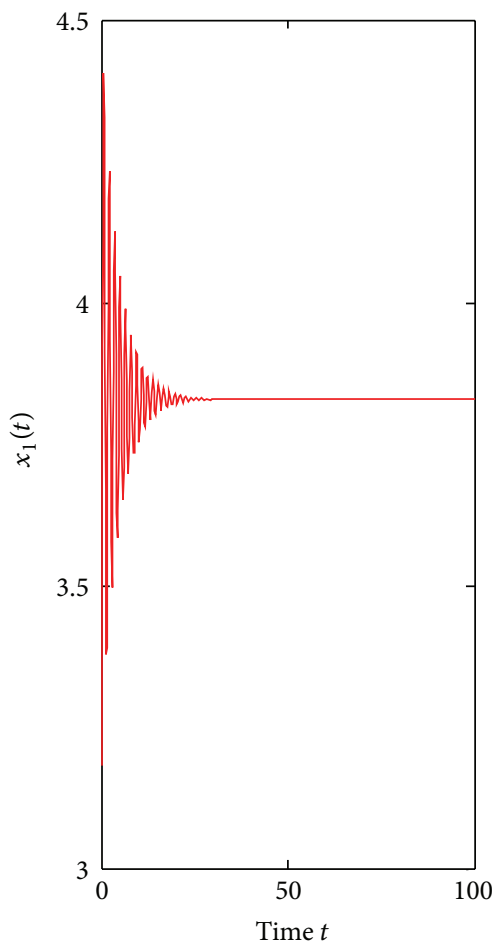

(a)

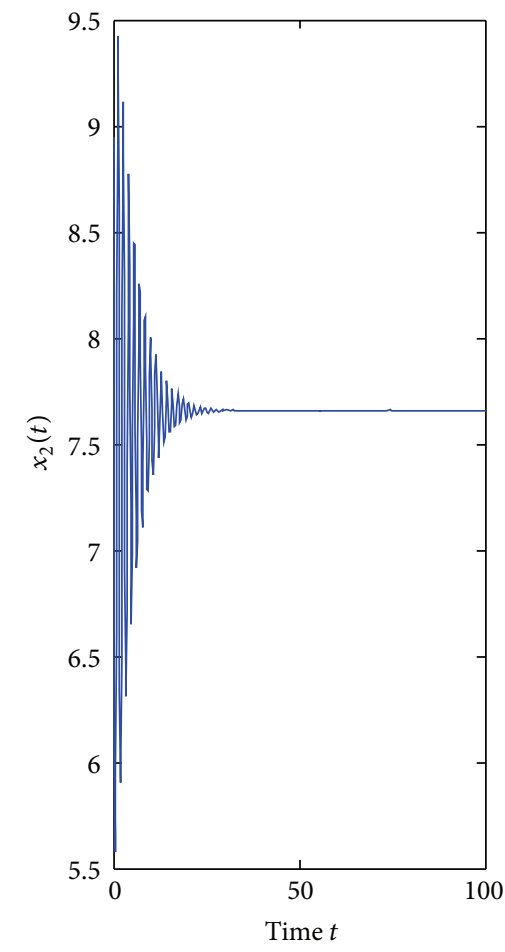

(b)

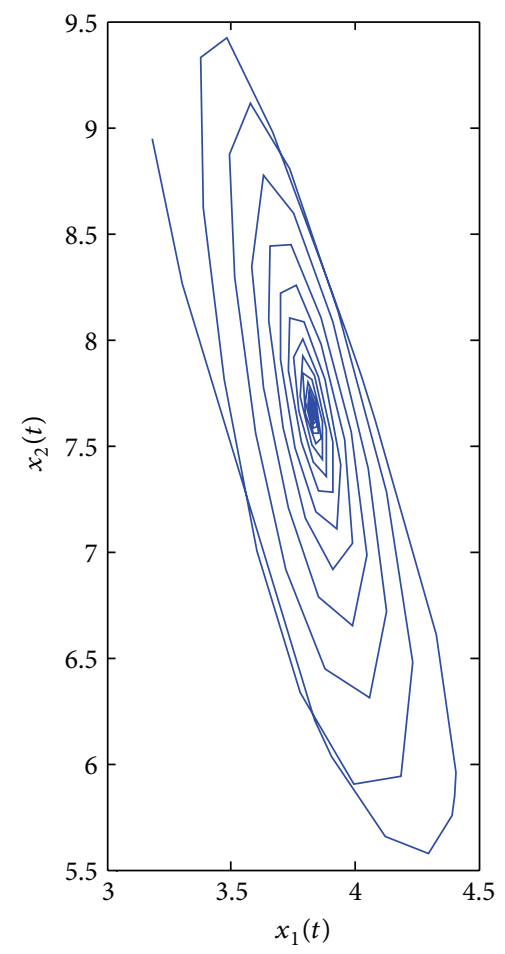

(c)

FigURE 7: $E^{*}$ is asymptotically stable for $\tau_{1}=0.325<\tau_{1 *}=0.3490$ at $\tau_{2}=0.3$.

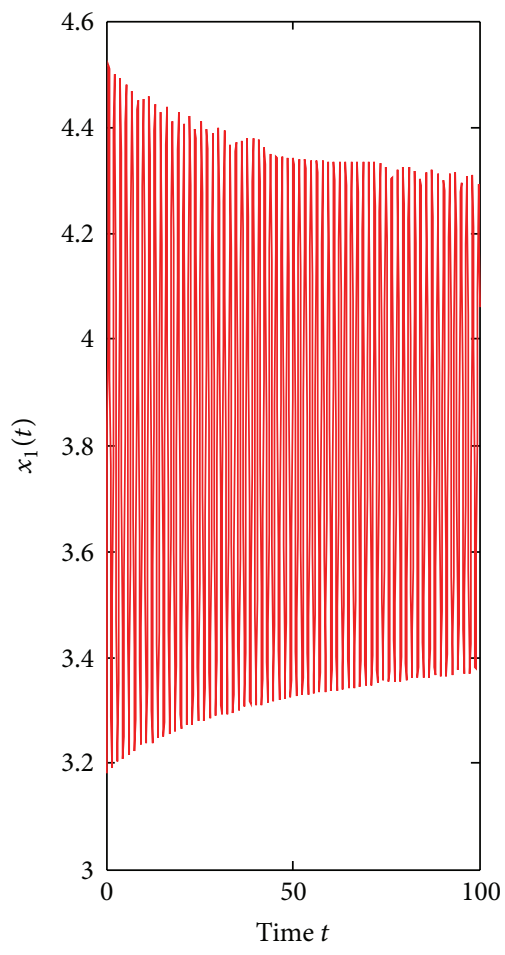

(a)

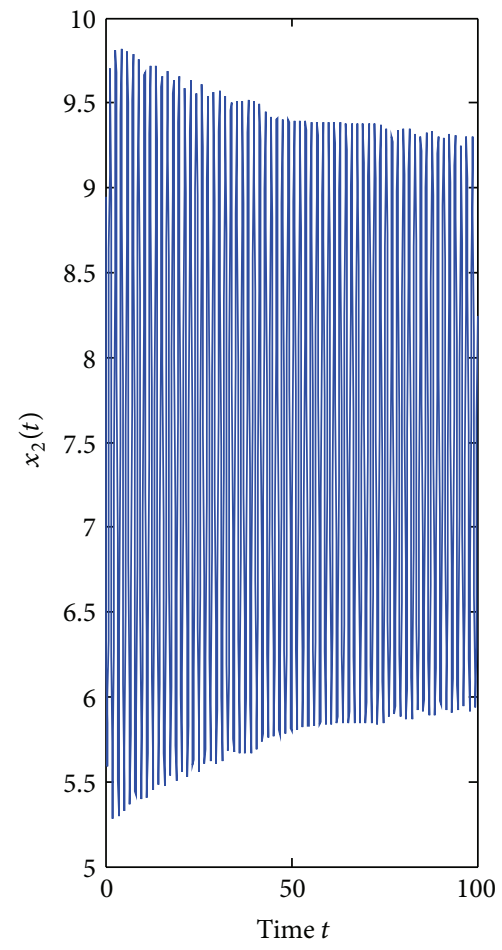

(b)

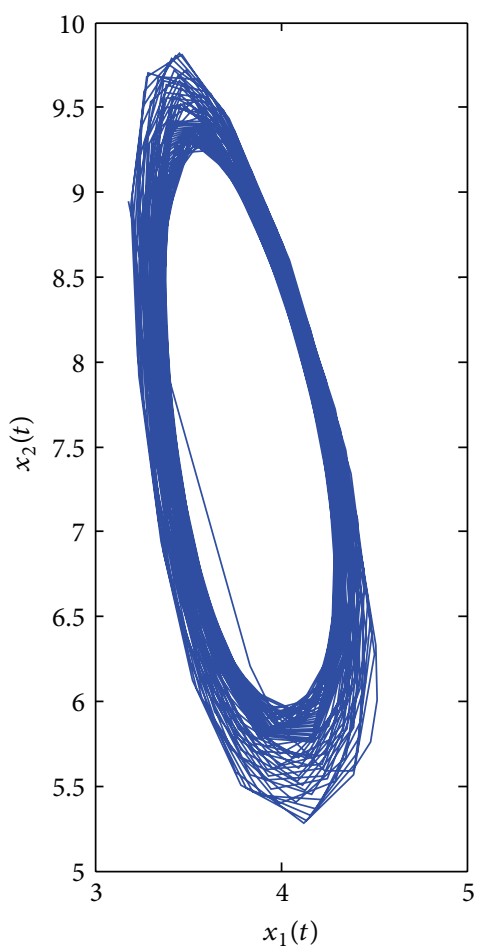

(c)

FIGURE 8: $E^{*}$ is unstable for $\tau_{1}=0.360>\tau_{1 *}=0.3490$ at $\tau_{2}=0.3$. 


$$
\begin{aligned}
& g_{02}=2 \tau_{1 *} \bar{D}\left[a_{13}+a_{14} \bar{q}^{(2)}(0)+a_{15} \bar{q}^{(1)}(-1)\right. \\
& +\bar{q}_{2}^{*}\left(c_{23}\left(\bar{q}^{(1)}\left(-\frac{\tau_{2 *}}{\tau_{1 *}}\right)\right)^{2}\right. \\
& +c_{24} \bar{q}^{(1)}\left(-\frac{\tau_{2 *}}{\tau_{1 *}}\right) \bar{q}^{(2)}(0) \\
& +c_{25} \bar{q}^{(2)}\left(-\frac{\tau_{2 *}}{\tau_{1 *}}\right) \bar{q}^{(2)}(0) \\
& \left.\left.+c_{26} \bar{q}^{(1)}\left(-\frac{\tau_{2 *}}{\tau_{1 *}}\right) \bar{q}^{(2)}\left(-\frac{\tau_{2 *}}{\tau_{1 *}}\right)\right)\right] \text {, } \\
& g_{21}=2 \tau_{1 *} \bar{D}\left[a_{13}\left(W_{11}^{(1)}(0)+W_{20}^{(1)}(0)\right)\right. \\
& +a_{14}\left(W_{11}^{(1)}(0) q^{(2)}(0)+\frac{1}{2} W_{20}^{(1)}(0) \bar{q}^{(2)}(0)\right. \\
& \left.+W_{11}^{(2)}(0)+\frac{1}{2} W_{20}^{(2)}(0)\right) \\
& +a_{15}\left(W_{11}^{(1)}(0) q^{(1)}(-1)+\frac{1}{2} W_{20}^{(1)}(0) \bar{q}^{(1)}(-1)\right. \\
& \left.+W_{11}^{(1)}(-1)+\frac{1}{2} W_{20}^{(1)}(-1)\right) \\
& +a_{16}\left(\bar{q}^{(2)}(0)+2 q^{(2)}(0)\right)+3 a_{17} \bar{q}^{(1)}(0) \\
& +\bar{q}_{2}^{*}\left(c _ { 2 3 } \left(2 W_{11}^{(1)}\left(-\frac{\tau_{2 *}}{\tau_{1 *}}\right) q^{(1)}\left(-\frac{\tau_{2 *}}{\tau_{1 *}}\right)\right.\right. \\
& \left.+W_{20}^{(1)}\left(-\frac{\tau_{2 *}}{\tau_{1 *}}\right) \bar{q}^{(1)}\left(-\frac{\tau_{2 *}}{\tau_{1 *}}\right)\right) \\
& +c_{24}\left(W_{11}^{(1)}\left(-\frac{\tau_{2 *}}{\tau_{1 *}}\right) q^{(2)}(0)\right. \\
& +\frac{1}{2} W_{20}^{(1)}\left(-\frac{\tau_{2 *}}{\tau_{1 *}}\right) \bar{q}^{(2)}(0) \\
& +W_{11}^{(2)}(0) q^{(1)}\left(-\frac{\tau_{2 *}}{\tau_{1 *}}\right) \\
& \left.+\frac{1}{2} W_{20}^{(2)}(0) \bar{q}^{(1)}\left(-\frac{\tau_{2 *}}{\tau_{1 *}}\right)\right) \\
& +c_{25}\left(W_{11}^{(2)}\left(-\frac{\tau_{2 *}}{\tau_{1 *}}\right) q^{(2)}(0)\right. \\
& +\frac{1}{2} W_{20}^{(2)}\left(-\frac{\tau_{2 *}}{\tau_{1 *}}\right) \bar{q}^{(2)}(0) \\
& +W_{11}^{(2)}(0) q^{(2)}\left(-\frac{\tau_{2 *}}{\tau_{1 *}}\right) \\
& \left.+\frac{1}{2} W_{20}^{(2)}(0) \bar{q}^{(2)}\left(-\frac{\tau_{2 *}}{\tau_{1 *}}\right)\right) \\
& +c_{26}\left(W_{11}^{(1)}\left(-\frac{\tau_{2 *}}{\tau_{1 *}}\right) q^{(2)}\left(-\frac{\tau_{2 *}}{\tau_{1 *}}\right)\right.
\end{aligned}
$$

$$
\begin{aligned}
& +\frac{1}{2} W_{20}^{(1)}\left(-\frac{\tau_{2 *}}{\tau_{1 *}}\right) \bar{q}^{(2)}\left(-\frac{\tau_{2 *}}{\tau_{1 *}}\right) \\
& +W_{11}^{(2)}\left(-\frac{\tau_{2 *}}{\tau_{1 *}}\right) q^{(1)}\left(-\frac{\tau_{2 *}}{\tau_{1 *}}\right) \\
& \left.+\frac{1}{2} W_{20}^{(2)}\left(-\frac{\tau_{2 *}}{\tau_{1 *}}\right) \bar{q}^{(1)}\left(-\frac{\tau_{2 *}}{\tau_{1 *}}\right)\right) \\
& +3 c_{27}\left(q^{(1)}\left(-\frac{\tau_{2 *}}{\tau_{1 *}}\right)\right)^{2} \bar{q}^{(1)}\left(-\frac{\tau_{2 *}}{\tau_{1 *}}\right) \\
& +c_{28}\left(\left(q^{(1)}\left(-\frac{\tau_{2 *}}{\tau_{1 *}}\right)\right)^{2} \bar{q}^{(2)}(0)\right. \\
& \left.+2 q^{(1)}\left(-\frac{\tau_{2 *}}{\tau_{1 *}}\right) q^{(2)}(0) \bar{q}^{(1)}\left(-\frac{\tau_{2 *}}{\tau_{1 *}}\right)\right) \\
& +c_{29}\left(\left(q^{(1)}\left(-\frac{\tau_{2 *}}{\tau_{1 *}}\right)\right)^{2} \bar{q}^{(2)}\left(-\frac{\tau_{2 *}}{\tau_{1 *}}\right)+2 q^{(1)}\right. \\
& \left.\times\left(-\frac{\tau_{2 *}}{\tau_{1 *}}\right) q^{(2)}\left(-\frac{\tau_{2 *}}{\tau_{1 *}}\right) \bar{q}^{(1)}\left(-\frac{\tau_{2 *}}{\tau_{1 *}}\right)\right) \\
& +c_{210}\left(q^{(1)}\left(-\frac{\tau_{2 *}}{\tau_{1 *}}\right) q^{(2)}\left(-\frac{\tau_{2 *}}{\tau_{1 *}}\right) \bar{q}^{(2)}(0)\right. \\
& +q^{(1)}\left(-\frac{\tau_{2 *}}{\tau_{1 *}}\right) \bar{q}^{(2)}\left(-\frac{\tau_{2 *}}{\tau_{1 *}}\right) \\
& \times q^{(2)}(0)+\bar{q}^{(1)}\left(-\frac{\tau_{2 *}}{\tau_{1 *}}\right) \\
& \left.\left.\left.\times q^{(2)}\left(-\frac{\tau_{2 *}}{\tau_{1 *}}\right) q^{(2)}(0)\right)\right)\right],
\end{aligned}
$$

with

$$
\begin{gathered}
W_{20}(\theta)=\frac{i g_{20} q(0)}{\omega_{1 *} \tau_{1 *}} e^{i \omega_{1 *} \tau_{1 *} \theta}+\frac{i \bar{g}_{02} \bar{q}(0)}{3 \omega_{1 *} \tau_{1 *}} e^{-i \omega_{1 *} \tau_{1 *} \theta} \\
+E_{1} e^{2 i \omega_{1 *} \tau_{1 *} \theta}, \\
W_{11}(\theta)=-\frac{i g_{11} q(0)}{\omega_{1 *} \tau_{1 *}} e^{i \omega_{1 *} \tau_{1 *} \theta}+\frac{i \bar{g}_{11} \bar{q}(0)}{\omega_{1 *} \tau_{1 *}} e^{-i \omega_{1 *} \tau_{1 *} \theta}+E_{2},
\end{gathered}
$$

where $E_{1}$ and $E_{2}$ can be determined by the following equations, respectively:

$$
\begin{gathered}
\left(\begin{array}{cc}
2 i \omega_{1 *}-a_{11}-b_{11} e^{-2 i \omega_{1 * \tau 1 *}} & -a_{12} \\
-c_{21} e^{-2 i \omega_{1 *} \tau_{2 *}} & 2 i \omega_{1 *}-c_{33} e^{-2 i \omega_{1 *} \tau_{2 *}}
\end{array}\right) E_{1} \\
=2\left(\begin{array}{c}
E_{1}^{(1)} \\
E_{1}^{(2)}
\end{array}\right), \\
\left(\begin{array}{cc}
a_{11}+b_{11} & a_{12} \\
c_{21} & c_{22}
\end{array}\right) E_{2}=-\left(\begin{array}{c}
E_{2}^{(1)} \\
E_{2}^{(2)}
\end{array}\right),
\end{gathered}
$$


with

$$
\begin{gathered}
E_{1}^{(1)}=a_{13}+a_{14} q^{(2)}(0)+a_{15} q^{(1)}(-1), \\
E_{1}^{(1)}=c_{23}\left(q^{(1)}\left(-\frac{\tau_{2 *}}{\tau_{1 *}}\right)\right)^{2}+c_{24} q^{(1)}\left(-\frac{\tau_{2 *}}{\tau_{1 *}}\right) q^{(2)}(0) \\
+c_{25} q^{(2)}\left(-\frac{\tau_{2 *}}{\tau_{1 *}}\right) q^{(2)}(0) \\
+c_{26} q^{(1)}\left(-\frac{\tau_{2 *}}{\tau_{1 *}}\right) q^{(2)}\left(-\frac{\tau_{2 *}}{\tau_{1 *}}\right), \\
E_{2}^{(1)}=2 a_{13}+a_{14}\left(q^{(2)}(0)+\bar{q}^{(2)}(0)\right) \\
+a_{15}\left(q^{(1)}(-1)+\bar{q}^{(1)}(-1)\right), \\
+c_{23} q^{(1)}\left(-\frac{\tau_{2 *}}{\tau_{1 *}}\right) \bar{q}^{(1)}\left(-\frac{\tau_{2 *}}{\tau_{1 *}}\right) \\
\left.+c_{25}^{(1)}\left(-\frac{\tau_{2 *}}{\tau_{1 *}}\right) \bar{q}^{(2)}(0)+\bar{q}^{(1)}\left(-\frac{\tau_{2 *}}{\tau_{1 *}}\right) q^{(2)}(0)\right) \\
+c_{26}\left(-\frac{\tau_{2 *}}{\tau_{1 *}}\right) \bar{q}^{(1)}\left(-\frac{\tau_{2 *}}{\tau_{1 *}}\right) \bar{q}^{(2)}\left(-\frac{\tau_{2 *}}{\tau_{1 *}}\right) \\
\left.+\bar{q}^{(1)}\left(-\frac{\tau_{2 *}}{\tau_{1 *}}\right) q^{(2)}\left(-\frac{\tau_{2 *}}{\tau_{1 *}}\right) q^{(2)}(0)\right) \\
\left.\tau_{1 *}\right)
\end{gathered}
$$

Then, we can get the following coefficients:

$$
\begin{gathered}
C_{1}(0)=\frac{i}{2 \omega_{1 *} \tau_{1 *}}\left(g_{11} g_{20}-2\left|g_{11}\right|^{2}-\frac{\left|g_{02}\right|^{2}}{3}\right)+\frac{g_{21}}{2}, \\
\mu_{2}=-\frac{\operatorname{Re}\left\{C_{1}(0)\right\}}{\operatorname{Re}\left\{\lambda^{\prime}\left(\tau_{1 *}\right)\right\}}, \\
\beta_{2}=2 \operatorname{Re}\left\{C_{1}(0)\right\}, \\
T_{2}=-\frac{\operatorname{Im}\left\{C_{1}(0)\right\}+\mu_{2} \operatorname{Im}\left\{\lambda^{\prime}\left(\tau_{1 *}\right)\right\}}{\omega_{1 *} \tau_{1 *}} .
\end{gathered}
$$

In conclusion, we have the following results.

Theorem 5. If $\mu_{2}>0\left(\mu_{2}<0\right)$, then the Hopf bifurcation is supercritical (subcritical); if $\beta_{2}<0\left(\beta_{2}>0\right)$, then the bifurcating periodic solutions are stable (unstable); if $T_{2}>0$ $\left(T_{2}<0\right)$, then the period of the bifurcating periodic solutions increases (decreases).

\section{Numerical Simulation and Discussions}

To demonstrate the algorithm for determining the existence of Hopf bifurcation in Section 2 and the properties of Hopf bifurcation in Section 3, we give an example of system (3) in the following form:

$$
\begin{gathered}
\frac{d x_{1}(t)}{d t}=x_{1}(t)\left[4-x_{1}\left(t-\tau_{1}\right)-\frac{0.5 x_{2}(t)}{4+x_{1}(t)+x_{1}^{2}(t)}\right], \\
\frac{d x_{2}(t)}{d t}=x_{2}(t)\left[3-\frac{1.5 x_{2}\left(t-\tau_{2}\right)}{x_{1}\left(t-\tau_{2}\right)}\right],
\end{gathered}
$$

which has a positive equilibrium $E^{*}(3.8298,7.6596)$. By calculation, we have $A+B+C=6.5788, D+E=11.2470$, and $D-E=-11.7318$. Namely, conditions $\left(H_{1}\right)$ and $\left(H_{2}\right)$ hold.

For $\tau_{1}>0, \tau_{2}=0$. We can get $\omega_{10}=3.9074$, $\tau_{10}=0.3788$. By Theorem 1, we know that when $\tau_{1} \in$ $\left[0, \tau_{10}\right)$, the positive equilibrium $E^{*}(3.8298,7.6596)$ is locally asymptotically stable and is unstable if $\tau_{1}>\tau_{10}$. As can be seen from Figure 1, when we let $\tau_{1}=0.35 \epsilon$ $\left[0, \tau_{10}\right)$, the positive equilibrium $E^{*}(3.8298,7.6596)$ is locally asymptotically stable. However, when $\tau_{1}=0.395>\tau_{10}$, the positive equilibrium $E^{*}(3.8298,7.6596)$ is unstable and a Hopf bifurcation occurs and a family of periodic solutions bifurcate from the positive equilibrium $E^{*}(3.8298,7.6596)$, which can be illustrated by Figure 2. Similarly, we can get $\omega_{20}=3.0827, \tau_{20}=0.4912$. The corresponding waveform and the phase plots are shown in Figures 3 and 4.

For $\tau_{1}=\tau_{2}=\tau>0$. We can obtain $\omega_{0}=$ 3.4601, $\tau_{0}=0.3361$. By Theorem 3, we know that when the time delay $\tau$ increases from zero to the critical value $\tau_{0}=0.3361$, the positive equilibrium $E^{*}(3.8298,7.6596)$ is locally asymptotically stable. It will lose its stability and a Hopf bifurcation occurs once $\tau>\tau_{0}=0.3361$. This property can be illustrated by Figures 5 and 6 .

Finally, consider $\tau_{1}$ as a parameter and let $\tau_{2}=0.3 \in$ $\left(0, \tau_{20}\right)$. We can get $\omega_{1 *}=2.3761, \tau_{1 *}=0.3490, \lambda^{\prime}\left(\tau_{1 *}\right)=$ $8.2230+2.6054 i$. From Theorem 4 , the positive equilibrium $E^{*}(3.8298,7.6596)$ is locally asymptotically stable for $\tau_{1} \epsilon$ $[0,0.3490)$ and unstable when $\tau_{1}>\tau_{1 *}=0.3490$, which can be seen from Figures 7 and 8 .

In addition, from (60), we can get $C_{1}(0)=-41.5020-$ 17.8366i. Furthermore, we have $\mu_{2}=5.0471>0, \beta_{2}=$ $-83.0040<0, T_{2}=5.6519>0$. Therefore, from Theorem 5 , we know that the Hopf bifurcation is supercritical. The bifurcating periodic solutions are stable and increase. If the bifurcating periodic solutions are stable, then the prey and the predator may coexist in an oscillatory mode. Therefore, the two species in system (3) may coexist in an oscillatory mode under certain conditions.

\section{Conflict of Interests}

The author declares that there is no conflict of interests regarding the publication of this paper.

\section{References}

[1] R. Arditi and L. R. Ginzburg, "Coupling in predator-prey dynamics: ratio-dependence," Journal of Theoretical Biology, vol. 139, no. 3, pp. 311-326, 1989. 
[2] R. Arditi, N. Perrin, and H. Saiah, "Functional responses and heterogeneities: an experimental test with cladocerans," Oikos, vol. 60, no. 1, pp. 69-75, 1991.

[3] I. Hanski, "The functional response of predators: worries about scale," Trends in Ecology and Evolution, vol. 6, no. 5, pp. 141-142, 1991.

[4] L. Zhang and C. Lu, "Periodic solutions for a semi-ratiodependent predator-prey system with Holling IV functional response," Journal of Applied Mathematics and Computing, vol. 32, no. 2, pp. 465-477, 2010.

[5] X. Ding, C. Lu, and M. Liu, "Periodic solutions for a semi-ratiodependent predator-prey system with nonmonotonic functional response and time delay," Nonlinear Analysis: Real World Applications, vol. 9, no. 3, pp. 762-775, 2008.

[6] X. Li and W. Yang, "Permanence of a semi-ratio-dependent predator-prey system with nonmonotonic functional response and time delay," Abstract and Applied Analysis, vol. 2009, Article ID 960823, 6 pages, 2009.

[7] M. Sen, M. Banerjee, and A. Morozov, "Bifurcation analysis of a ratio-dependent prey-predator model with the Allee effect," Ecological Complexity, vol. 11, pp. 12-27, 2012.

[8] X. Ding and G. Zhao, "Periodic solutions for a semi-ratiodependent predator-prey system with delays on time scales," Discrete Dynamics in Nature and Society, vol. 2012, Article ID 928704, 15 pages, 2012.

[9] B. Yang, "Pattern formation in a diffusive ratio-dependent Holling-Tanner predator-prey model with Smith growth," Discrete Dynamics in Nature and Society, vol. 2013, Article ID 454209, 8 pages, 2013.

[10] Z. Yue and W. Wang, "Qualitative analysis of a diffusive ratiodependent Holling-Tanner predator-prey model with Smith growth," Discrete Dynamics in Nature and Society, vol. 2013, Article ID 267173, 9 pages, 2013.

[11] M. Haque, "Ratio-dependent predator-prey models of interacting populations," Bulletin of Mathematical Biology, vol. 71, no. 2, pp. 430-452, 2009.

[12] M. Ferrara and L. Guerrini, "Center manifold analysis for a delayed model with classical saving," Far East Journal of Mathematical Sciences, vol. 70, no. 2, pp. 261-269, 2012.

[13] S. Guo and W. Jiang, "Hopf bifurcation analysis on general Gause-type predator-prey models with delay," Abstract and Applied Analysis, vol. 2012, Article ID 363051, 17 pages, 2012.

[14] C. Y. Wang, S. Wang, F. P. Yang, and L. R. Li, "Global asymptotic stability of positive equilibrium of three-species Lotka-Volterra mutualism models with diffusion and delay effects," Applied Mathematical Modelling, vol. 34, no. 12, pp. 4278-4288, 2010.

[15] M. Ferrara, L. Guerrini, and R. Mavilia, "Modified neoclassical growth models with delay: a critical survey and perspectives," Applied Mathematical Sciences, vol. 7, pp. 4249-4257, 2013.

[16] J. J. Jiao, L. S. Chen, and J. J. Nieto, "Permanence and global attractivity of stage-structured predator-prey model with continuous harvesting on predator and impulsive stocking on prey," Applied Mathematics and Mechanics, vol. 29, no. 5, pp. 653-663, 2008.

[17] Y. Zhu and K. Wang, "Existence and global attractivity of positive periodic solutions for a predator-prey model with modified Leslie-Gower Holling-type II schemes," Journal of Mathematical Analysis and Applications, vol. 384, no. 2, pp. 400408, 2011.

[18] M. Ferrara, L. Guerrini, and C. Bianca, “The Cai model with time delay: existence of periodic solutions and asymptotic analysis," Applied Mathematics \& Information Sciences, vol. 7, no. 1, pp. 21-27, 2013.

[19] R. M. Etoua and C. Rousseau, "Bifurcation analysis of a generalized Gause model with prey harvesting and a generalized Holling response function of type III," Journal of Differential Equations, vol. 249, no. 9, pp. 2316-2356, 2010.

[20] Y. Yang, "Hopf bifurcation in a two-competitor, one-prey system with time delay," Applied Mathematics and Computation, vol. 214, no. 1, pp. 228-235, 2009.

[21] Y. Qu and J. Wei, "Bifurcation analysis in a time-delay model for prey-predator growth with stage-structure," Nonlinear Dynamics, vol. 49, no. 1-2, pp. 285-294, 2007.

[22] B. D. Hassard, N. D. Kazarinoff, and Y. H. Wan, Theory and Applications of Hopf Bifurcation, Cambridge University Press, Cambridge, UK, 1981. 


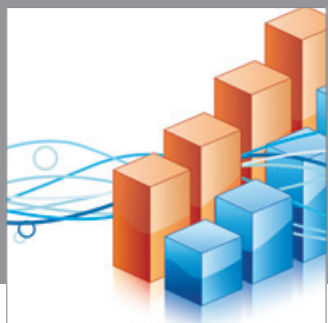

Advances in

Operations Research

mansans

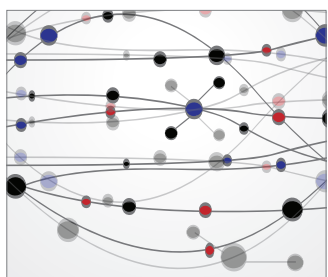

The Scientific World Journal
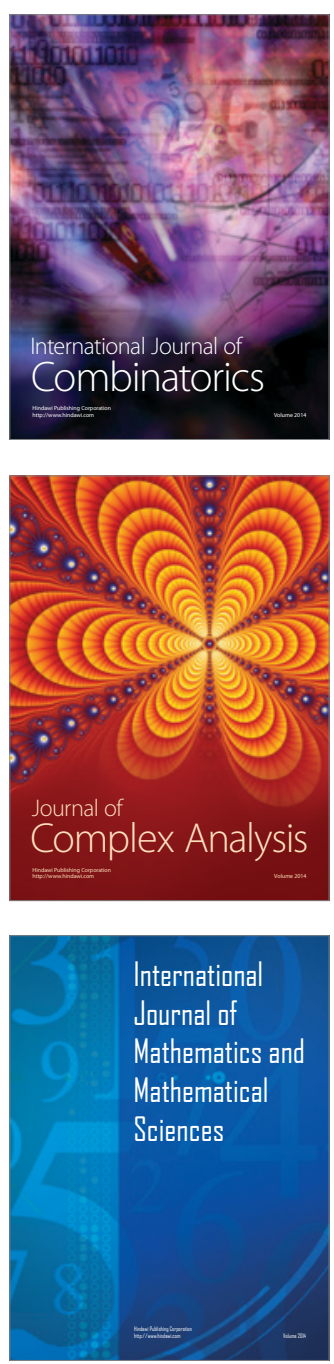
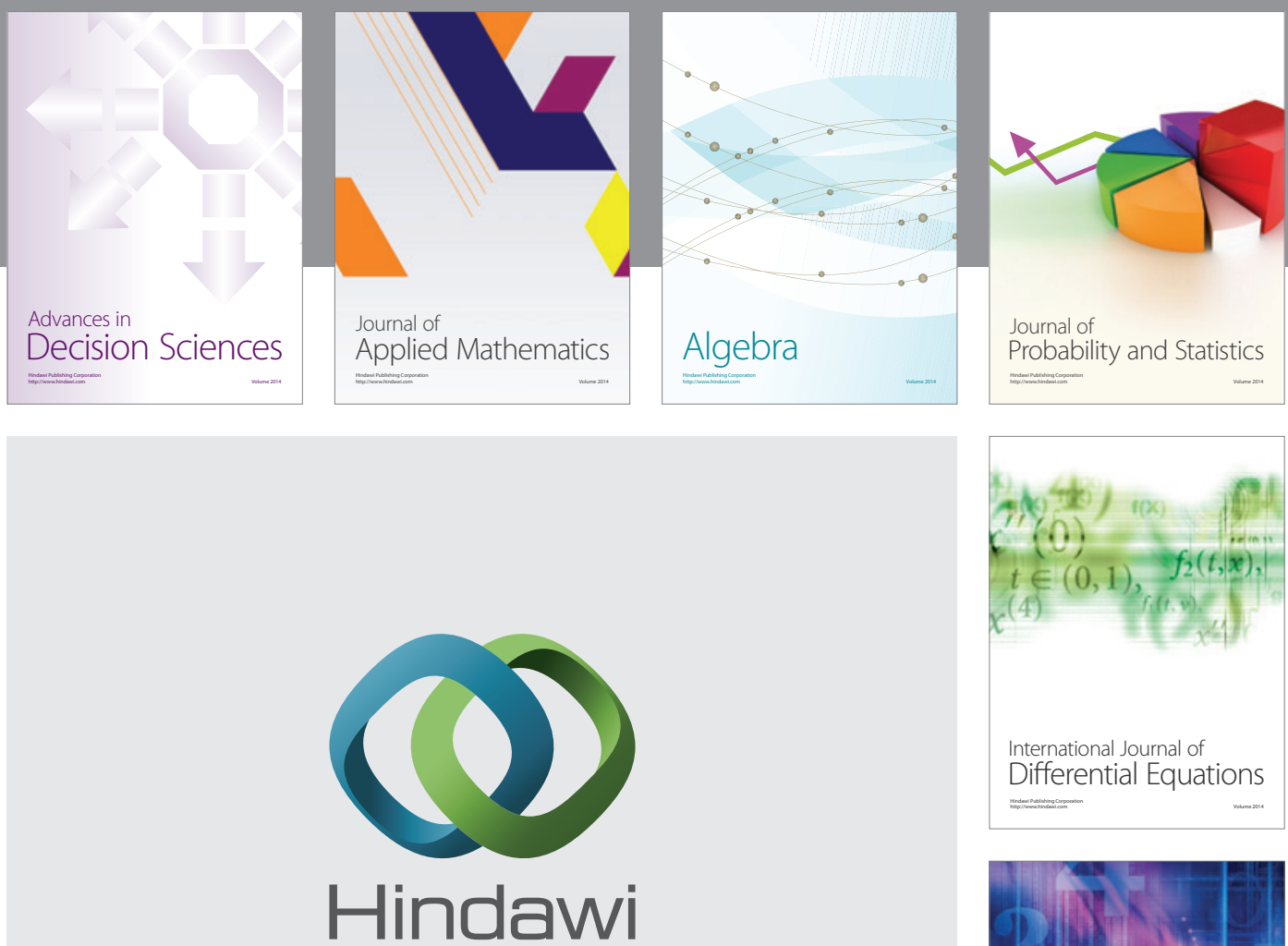

Submit your manuscripts at http://www.hindawi.com
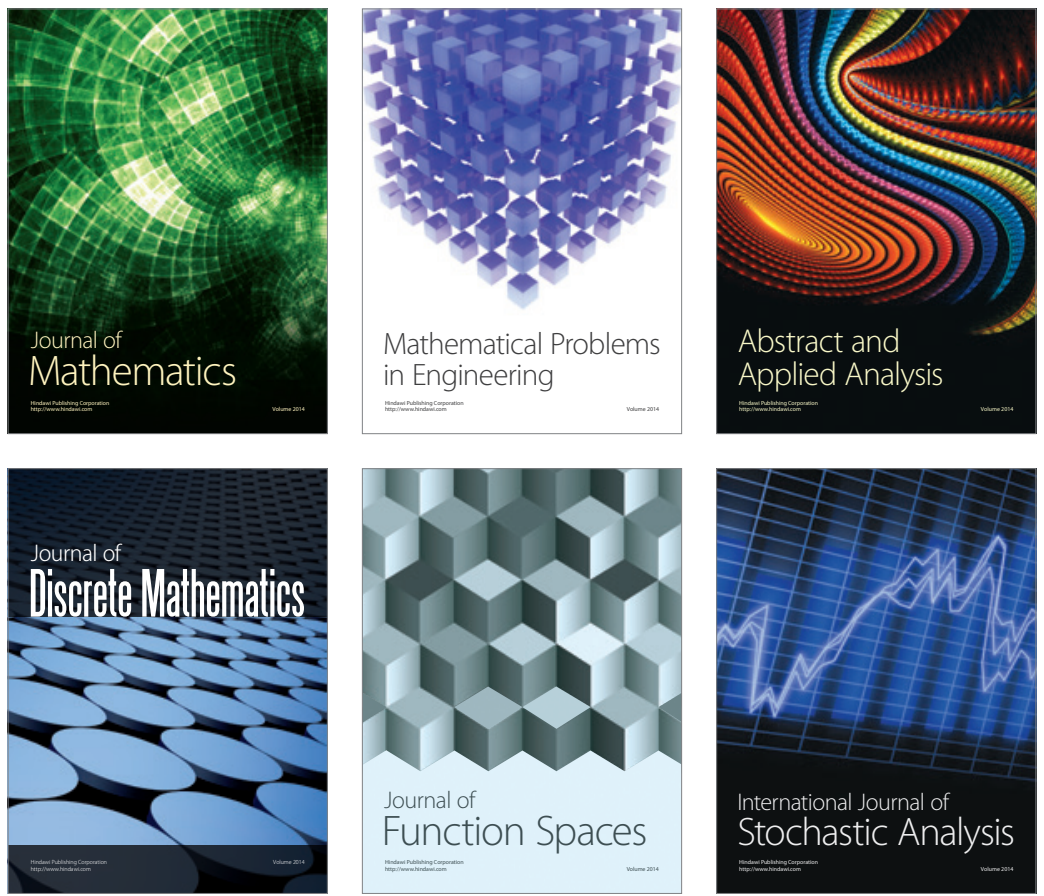

Journal of

Function Spaces

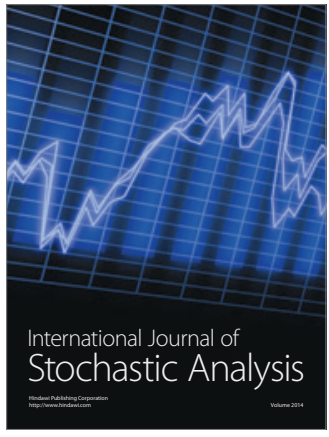

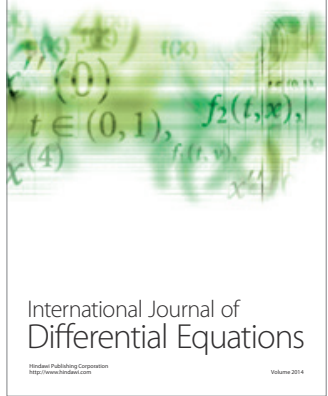
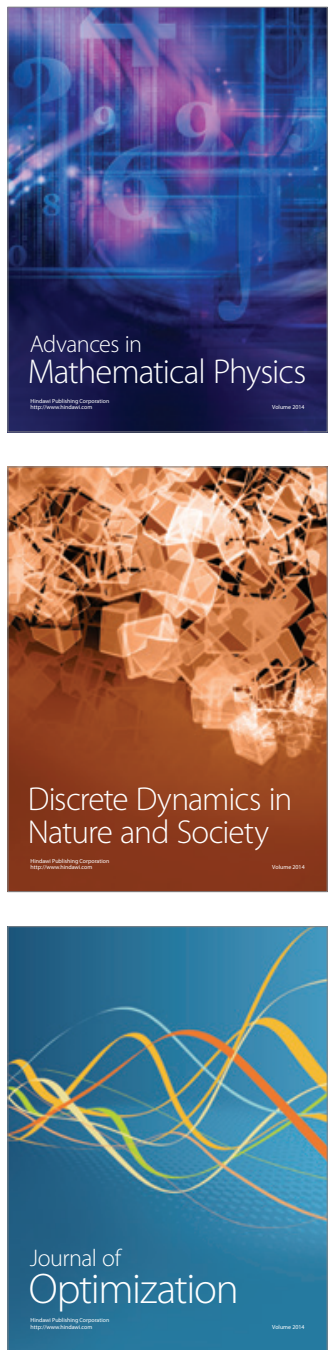